\title{
Vapor wall deposition in Teflon chambers
}

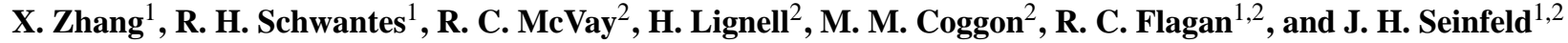 \\ ${ }^{1}$ Division of Engineering and Applied Science, California Institute of Technology, Pasadena, CA, USA \\ ${ }^{2}$ Division of Chemistry and Chemical Engineering, California Institute of Technology, Pasadena, CA, USA
}

Correspondence to: J. H. Seinfeld (seinfeld@caltech.edu)

Received: 23 September 2014 - Published in Atmos. Chem. Phys. Discuss.: 24 October 2014

Revised: 7 February 2015 - Accepted: 25 March 2015 - Published: 23 April 2015

Abstract. Teflon chambers are ubiquitous in studies of atmospheric chemistry. Secondary organic aerosol (SOA) formation can be underestimated, owing to deposition of SOAforming vapors to the chamber wall. We present here an experimental protocol and a model framework to constrain the vapor-wall interactions in Teflon chambers. We measured the wall deposition rates of 25 oxidized organic compounds generated from the photooxidation of isoprene, toluene, $\alpha$ pinene, and dodecane in two chambers that had been extensively used and in two new unused chambers. We found that the extent of prior use of the chamber did not significantly affect the sorption behavior of the Teflon films. Among the 25 compounds studied, the maximum wall deposition rate is exhibited by the most highly oxygenated and least volatile compounds. By optimizing the model output to the observed vapor decay profiles, we identified that the dominant parameter governing the extent of wall deposition of a compound is its wall accommodation coefficient $\left(\alpha_{\mathrm{w}, i}\right)$, which can be correlated through its volatility with the number of carbons and oxygens in the molecule. By doing so, the wall-induced deposition rate of intermediate/semi-volatile organic vapors can be reasonably predicted based on their molecular constituency. The extent to which vapor wall deposition impacts measured SOA yields depends on the competition between uptake of organic vapors by suspended particles and the chamber wall. The timescale associated with vapor wall deposition can vary from minutes to hours depending on the value of $\alpha_{\mathrm{w}, i}$. For volatile and intermediate volatility organic compounds (small $\alpha_{\mathrm{w}, i}$ ), gas-particle partitioning will dominate wall deposition for typical particle number concentrations in chamber experiments. For compounds characterized by relatively large $\alpha_{\mathrm{w}, i}$, vapor transport to particles is suppressed by competition with the chamber wall even with perfect particle accommodation.

\section{Introduction}

Understanding of the mechanism and extent of secondary organic aerosol (SOA) formation from oxidation of volatile organic compounds (VOCs) has been derived largely from experiments in Teflon chambers. Chamber-measured SOA yields (mass of SOA formed per mass of VOC reacted) have been widely parameterized into regional/global atmospheric models, and chemical mechanisms leading to SOA formation and aging have been derived based on the gas/particlephase identification of intermediate/semi/low-volatility compounds generated in controlled chamber experiments. An unavoidable consequence of the use of an environmental chamber is interaction of vapors and particles with the chamber wall. It has been recently established that SOA formation can be substantially underestimated due to deposition of SOAforming vapors to the chamber wall rather than growing particles (Zhang et al., 2014a).

Chamber-wall-induced decay of organic vapors was reported 30 years ago. Grosjean (1985) and McMurry and Grosjean (1985) measured wall deposition rates of several volatile organic compounds in a chamber constructed from Fluorinated ethylene propylene (FEP) Teflon film. The lifetime of the VOCs, with respect to wall deposition, was found generally to exceed $\sim 15 \mathrm{~h}$. Loza et al. (2010) found that deposition of the isoprene oxidation product surrogate, 2,3-epoxy-1,4-butanediol (BEPOX), and glyoxal to the FEP Teflon chamber wall is reversible on sufficiently long timescales. On the contrary, rapid reversible gas-wall partitioning of $n$-alkanes, 1-alkenes, 2-alcohols, 2 ketones, monoacids, and 1,2-diols was universally observed by Matsunaga and Ziemann (2010) and Yeh and Ziemann (2014). Following the same experimental protocol, Kokkola et al. (2014) measured that the equilibrium fractions of 


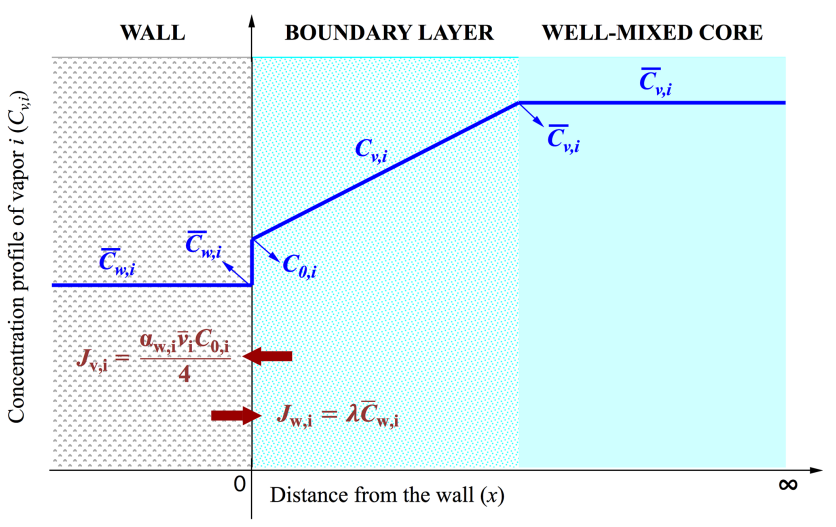

Figure 1. Theoretical framework representing the vapor-wall interactions. Concentrations of organic vapor $i$ in the well-mixed core, in the boundary layer, over the surface of the chamber wall, and in the chamber wall are denoted by $\bar{C}_{\mathrm{v}, i}, C_{\mathrm{v}, i}, C_{0, i}, \bar{C}_{\mathrm{w}, i}$, respectively. Vapor fluxes at the gas-wall interface are denoted by $J_{\mathrm{v}, i}$ and $J_{\mathrm{w}, i}$.

nopinone and pinanediol on the wall of a $4 \mathrm{~m}^{3}$ FEP Teflon chamber are on average 0.4 and 0.8 , respectively.

The extent to which vapors and the chamber wall interact is reflected by properties such as the gas-phase transport rate of organic molecules, affinity of the wall for various organic molecules, the degree of reversibility of the vapor-wall partitioning, and the equilibrium solubility of organic vapors in the wall. Organic materials generated in chamber experiments can deposit on the chamber wall to form a coating that can act as the primary absorbing medium, or the Teflon film itself could act as the absorbing medium, in a process akin to the sorption of small molecules by organic polymers. While measurement of vapor wall deposition rates for the thousands of organic molecules that are produced from the oxidation of SOA precursor VOCs is not presently possible, empirical expressions that represent the deposition rates of organic vapors as a function of general molecular properties would be highly useful.

A prime goal of characterizing vapor wall deposition in a chamber is to understand its impact on SOA formation and evolution. We present here an experimental protocol to constrain the nature of organic vapor wall deposition in Teflon chambers. We measured wall-induced dark decay rates of 25 intermediate/semi-volatility organic vapors, which span a range of volatilities and oxidation states, in both unused and previously used chambers constructed with FEP Teflon film. A temperature ramping program $(298-318 \mathrm{~K})$ was implemented to study the reversibility of vapor-wall partitioning. A model framework is developed to describe interactions between organic vapors and the chamber wall following the theories for particle wall deposition and gas-particle partitioning. We address the following questions in the present study. (1) What is the physicochemical nature of the chamber wall? (2) What are the key parameters that characterize the vapor-wall interactions and how can these values be de- termined? (3) How can one predict the wall deposition rate of a specific compound based on its molecular properties?

\section{Vapor wall deposition - theory}

Figure 1 depicts the steady-state concentration profiles of an organic compound $i$ in the well-mixed core of the chamber $\left(\bar{C}_{\mathrm{v}, i}\right)$, in the boundary layer adjacent to the wall $\left(C_{\mathrm{v}, i}\right)$, at the wall surface $\left(C_{0, i}\right)$, and in the chamber wall $\left(\bar{C}_{\mathrm{w}, i}\right)$. Vapor molecules in the well-mixed core of a chamber are transported through a boundary layer adjacent to the wall by a combination of molecular and turbulent diffusion. The transport rate depends on both the molecular properties of the individual organic compound (as characterized by the molecular diffusion coefficient, $D_{i}$ ), as well as the extent of turbulent mixing in the chamber (as characterized by the coefficient of eddy diffusion, $K_{\mathrm{e}}$ ). As vapor molecules encounter the chamber wall, the fraction of those encounters that lead to uptake is represented by the accommodation coefficient $\left(\alpha_{\mathrm{w}, i}\right)$, and molecules rebound with a probability of $1-\alpha_{\mathrm{w}, i}$. The accommodation coefficient depends, in principle, on the nature of the wall surface as well as the compound chemical composition. It is worth emphasizing that $\alpha_{\mathrm{w}, i}$ characterizes imperfect wall accommodation of the gas-wall interface. Molecules deposited on the wall may re-evaporate at a rate that depends on their concentration in the wall. In order to represent this process, we note that, at equilibrium, the flux arriving from the gas phase $\left(J_{\mathrm{v}, i}\right)$ and the evaporation flux from the wall $\left(J_{\mathrm{w}, i}\right)$ are equal. Thus, the evaporative flux from the wall $\left(J_{\mathrm{w}, i}\right)$ can be expressed as a function of the accommodation coefficient $\left(\alpha_{\mathrm{w}, i}\right)$, as described in Eqs. (7)-(9) later.

A conservation balance on $\bar{C}_{\mathrm{v}, i}$, the concentration of vapor $i$ in the well-mixed core of a chamber that is subject only to the deposition process, is given by

$\frac{d \bar{C}_{\mathrm{v}, i}}{\mathrm{~d} t}=-k_{\mathrm{w}, \text { depo }, i} \bar{C}_{\mathrm{v}, i}+k_{\mathrm{w}, \text { evap }, i} \bar{C}_{\mathrm{w}, i}$,

where $k_{\mathrm{w}, \text { depo, } i}\left(\mathrm{~s}^{-1}\right)$ is the deposition rate coefficient to the wall, $k_{\mathrm{w}, \text { evap }, i}\left(\mathrm{~s}^{-1}\right)$ is the evaporation rate coefficient from the wall, and $\bar{C}_{\mathrm{w}, i}$ is the concentration of vapor $i$ that has accumulated on the chamber wall. The dynamic behavior of $\bar{C}_{\mathrm{w}, i}$ is described by a corresponding balance:

$\frac{d \bar{C}_{\mathrm{w}, i}}{\mathrm{~d} t}=-k_{\mathrm{w}, \text { evap }, i} \bar{C}_{\mathrm{w}, i}+k_{\mathrm{w}, \text { depo }, i} \bar{C}_{\mathrm{v}, i}$.

Note that $\bar{C}_{\mathrm{w}, i}$ is assumed to be zero at the onset of vapor $i$ generation, ultimately reaching equilibrium with $\bar{C}_{\mathrm{v}, i}$.

\subsection{Vapor flux arriving from the gas phase $\left(J_{\mathrm{v}, i}\right)$}

For a chamber that is relatively well mixed, transport to the wall occurs by molecular and turbulent diffusion across a thin boundary layer, of thickness $\delta$, adjacent to the chamber wall. 
The flux due to molecular diffusion is given by $-D_{i} \nabla C_{\mathrm{v}, i}$, where $C_{\mathrm{V}, i}$ is the local vapor $i$ concentration in the boundary layer and $D_{i}$ is its molecular diffusivity. The turbulent diffusion flux is expressed as $-D_{\mathrm{e}} \nabla C_{\mathrm{v}, i}$, where $D_{\mathrm{e}}$ is the eddy diffusivity. One can invoke the Prandtl mixing length expression near a wall, $D_{\mathrm{e}}=K_{\mathrm{e}} x^{2}$, where $x$ is the distance from the wall, and $K_{\mathrm{e}}$ is the coefficient of eddy diffusion (Corner and Pendlebury, 1951; Crump and Seinfeld, 1981). Owing to the small value of $\delta$, a quasi-steady state condition exists in the boundary layer, and the concentration of vapor $i$ within the boundary layer, $0 \leq x \leq \delta$, is governed by

$\frac{d}{\mathrm{~d} x}\left[\left(K_{\mathrm{e}} x^{2}+D_{i}\right) \frac{d C_{\mathrm{v}, i}}{\mathrm{~d} x}\right]=0$.

Introducing the dimensionless variable $z$ by setting $x=$ $\left(D_{i} / K_{\mathrm{e}}\right)^{1 / 2} z$, Eq. (3) becomes

$$
\left(z^{2}+1\right) \frac{d^{2} C_{\mathrm{v}, i}}{\mathrm{~d} z^{2}}+2 z \frac{\mathrm{d} C_{\mathrm{v}, i}}{\mathrm{~d} z}=0,
$$

subject to the boundary conditions,

$x=0(z=0) \rightarrow C_{\mathrm{v}, i}=C_{0, i}$,

$x=\delta\left(z=\left(K_{\mathrm{e}} / D_{i}\right)^{1 / 2} \delta\right) \rightarrow C_{\mathrm{v}, i}=\bar{C}_{\mathrm{v}, i}$,

where $C_{0, i}$ and $\bar{C}_{\mathrm{v}, i}$ are concentrations of vapor $i$ over the wall surface and in the well-mixed core of the chamber, respectively. Note that the accommodation coefficient for particles on the wall was assumed to be unity in previous theoretical studies (e.g., Crump and Seinfeld, 1981; McMurry and Grosjean, 1985), meaning that particles that encounter the wall will lead to $100 \%$ uptake. This assumption is reasonable, especially if particles are in a quasi-liquid state. On the other hand, the accommodation coefficient for vapors on the wall $\left(\alpha_{\mathrm{w}, i}\right)$ is likely less than unity, and the steady-state concentration is then nonzero at the chamber wall surface. The solution of Eq. (4) expressed in the original variables is

$$
\begin{aligned}
C_{\mathrm{v}, i} & =C_{0, i}+\left(\bar{C}_{\mathrm{v}, i}-C_{0, i}\right) \frac{\tan ^{-1}\left[\left(K_{\mathrm{e}} / D_{i}\right)^{1 / 2} x\right]}{\tan ^{-1}\left[\left(K_{\mathrm{e}} / D_{i}\right)^{1 / 2} \delta\right]} \\
& \approx C_{0, i}+\left(\bar{C}_{\mathrm{v}, i}-C_{0, i}\right) \frac{\tan ^{-1}\left[\left(K_{\mathrm{e}} / D_{i}\right)^{1 / 2} x\right]}{\pi / 2} .
\end{aligned}
$$

Physically, turbulent diffusion dominates molecular diffusion at the outer edge of the boundary layer, so that $\left(K_{\mathrm{e}} / D_{i}\right)^{1 / 2} \delta \gg 1$.

The vapor flux arriving from the gas phase to the wall surface $\left(J_{\mathrm{v}, i}\right)$ is derived from the kinetic theory of gases:

$J_{\mathrm{v}, i}=\frac{\alpha_{\mathrm{w}, i} \bar{v}_{i} C_{0, i}}{4}$,

where $\bar{v}_{i}$ is the species mean thermal speed.

\subsection{Vapor flux leaving from the wall due to evaporation $\left(J_{\mathrm{w}, i}\right)$}

Without loss of generality, vapor wall deposition can be assumed to be reversible. The flux of molecules $i$ that evaporate from the wall back to the gas phase $\left(J_{\mathrm{w}, i}\right)$ depends on the concentration of $i$ in the wall $\left(\bar{C}_{\mathrm{w}, i}\right)$. So we can write $J_{\mathrm{w}, i}$ as a function of $\bar{C}_{\mathrm{w}, i}$ :

$J_{\mathrm{w}, i} \propto \bar{C}_{\mathrm{w}, i}$ or $J_{\mathrm{w}, i}=\lambda \bar{C}_{\mathrm{w}, i}$,

where $\lambda$ is simply a quantity that reflects the positive correlation between $J_{\mathrm{w}, i}$ and $\bar{C}_{\mathrm{w}, i}$. If the gas and wall phases are at equilibrium, then

$J_{\mathrm{v}, i,(\mathrm{eq})}=J_{\mathrm{w}, i,(\mathrm{eq})}$.

Therefore,

$\lambda=\frac{\alpha_{\mathrm{w}, i} \bar{v}_{i} C_{0, \mathrm{i}, \mathrm{eq}}}{4 \bar{C}_{\mathrm{w}, i, \mathrm{eq}}}=\frac{\alpha_{\mathrm{w}, i} \bar{v}_{i}}{4 H_{i}}$,

where $H_{i}$ is the Henry's law constant of organic species $i$. Substitution of Eq. (9) into Eq. (7) gives

$J_{\mathrm{w}, i}=\frac{\alpha_{\mathrm{w}, i} \bar{v}_{i} \bar{C}_{\mathrm{w}, i}}{4 H_{i}}$.

If applying vapor-particle partitioning theory here, Eq. (10) can be rewritten as

$J_{\mathrm{w}, i}=\frac{\alpha_{\mathrm{w}, i} \bar{v}_{i} \bar{C}_{\mathrm{w}, i}}{4 K_{\mathrm{w}, i} C_{\mathrm{w}}}$,

where $K_{\mathrm{w}, i}$ is vapor-wall partition coefficient (Matsunaga and Ziemann, 2010):

$K_{\mathrm{w}, i}=\frac{R T}{p_{\mathrm{L}, i}^{0} \gamma_{i} \bar{M}_{\mathrm{w}}}$,

and where $p_{\mathrm{L}, i}^{0}$ is the vapor pressure of compound $i$ as a liquid. We calculate $p_{\mathrm{L}, i}^{0}$ by the average of two group contribution methods, "SIMPOL.1" developed by Pankow and Asher (2008) and "EVAPORATION" developed by Compernolle et al. (2011). $\gamma_{i}$, the activity coefficient in the wall layer on a mole fraction basis, is assumed to be unity here, $R$ is the gas constant, $T$ is temperature, and $\bar{M}_{\mathrm{w}}$ is the average molecular weight of the absorbing organic material on the wall, which, following Matsunaga and Ziemann (2010), is assumed to be $250 \mathrm{~g} \mathrm{~mol}^{-1} \cdot C_{\mathrm{W}}\left(\mathrm{g} \mathrm{m}^{-3}\right)$ is an assumed equivalent mass of absorbing organic material on the chamber wall (Matsunaga and Ziemann, 2010). It can be regarded as characterizing the equilibrium solubility of individual organic molecules in FEP Teflon polymer and, possibly, in other organic materials deposited on the wall. When $C_{\mathrm{w}} \rightarrow \infty$, the wall presents essentially an absorbing medium of infinite extent, and vapor wall deposition is ultimately an irreversible process. Note, 
however, that the concept of an "equivalent absorbing organic mass" does not necessarily imply that an actual layer of organic material exists on the chamber wall. $C_{\mathrm{w}}$ might well represent the accumulation of deposited organic material from previous chamber experiments, or it could reflect the absorption properties of FEP film itself. We will return to the nature of $C_{\mathrm{w}}$ shortly.

Since the gas-wall interface is presumed to have no thickness, the net flux across the interface results from the concentration gradient,

$\left.D_{i} \frac{\mathrm{d} C_{\mathrm{v}, i}}{\mathrm{~d} x}\right|_{x=0}=J_{\mathrm{v}, i}-J_{\mathrm{w}, i}=\frac{\alpha_{\mathrm{w}, i} \bar{v}_{i} C_{0, i}}{4}-\frac{\alpha_{\mathrm{w}, i} \bar{v}_{i} \bar{C}_{\mathrm{w}, i}}{4 K_{\mathrm{w}, i} C_{\mathrm{w}}}$.

Note that when equilibrium is established, the net flux becomes zero and the concentration gradient no longer exists at the gas-wall interface. The LHS of Eq. (13) is based on Fick's law of diffusion and leads to Eq. (5). In this way, the quantity $C_{0, i}$ is expressed as a function of $\bar{C}_{\mathrm{v}, i}$ and $\bar{C}_{\mathrm{w}, i}$. Therefore, the conservation equation for the change in the concentration of vapor $i$ in the well-mixed core of the chamber owing to wall deposition is given by

$\frac{d \bar{C}_{\mathrm{v}, i}}{\mathrm{~d} t}=\left(\frac{A}{V}\right)\left(\frac{\alpha_{\mathrm{w}, i} \bar{v}_{i} / 4}{\pi \alpha_{\mathrm{w}, i} \bar{v}_{i} / 8\left(D_{i} K_{\mathrm{e}}\right)^{1 / 2}+1}\right)\left(\frac{\bar{C}_{\mathrm{w}, i}}{K_{\mathrm{w}, i} C_{\mathrm{w}}}-\bar{C}_{\mathrm{v}, i}\right)$,

where $A$ and $V$ are the surface area and volume of the chamber, respectively. A rewrite of Eq. (14) gives

$k_{\mathrm{w}, \mathrm{depo}, i}=\left(\frac{A}{V}\right)\left(\frac{\alpha_{\mathrm{w}, i} \bar{v}_{i} / 4}{\pi \alpha_{\mathrm{w}, i} \bar{v}_{i} / 8\left(D_{i} K_{\mathrm{e}}\right)^{1 / 2}+1}\right)$,

$k_{\mathrm{w}, \text { evap }, i}=\frac{k_{\mathrm{w}, \text { depo }, i}}{K_{\mathrm{w}, i} C_{\mathrm{w}}}$.

\section{Vapor wall deposition - experiment}

Experiments were conducted in the Caltech dual $24 \mathrm{~m}^{3}$ Fluorinated ethylene propylene (FEP) Teflon chambers that are suitable for pristine (low-NO) and polluted (high-NO) conditions (Zhang and Seinfeld, 2013; Fahnestock et al., 2014; Loza et al., 2014). Figure 2 shows a schematic of the experimental protocol used to measure deposition of organic vapors to the chamber wall. Oxidized organic vapors were generated via photooxidation of four parent VOCs, isoprene, toluene, $\alpha$-pinene, and dodecane, in the absence of seed aerosol. Once a sufficient amount of oxidized products is formed with none or limited aerosol formation via nucleation, irradiation is ceased, and the ensuing wall-induced dark decay of the array of oxidation products is monitored by chemical ionization mass spectrometry (CIMS). Following this period, the chambers were heated to investigate the extent to which vaporwall partitioning is reversible. These experiments were carried out in two chambers that had been used in past SOA

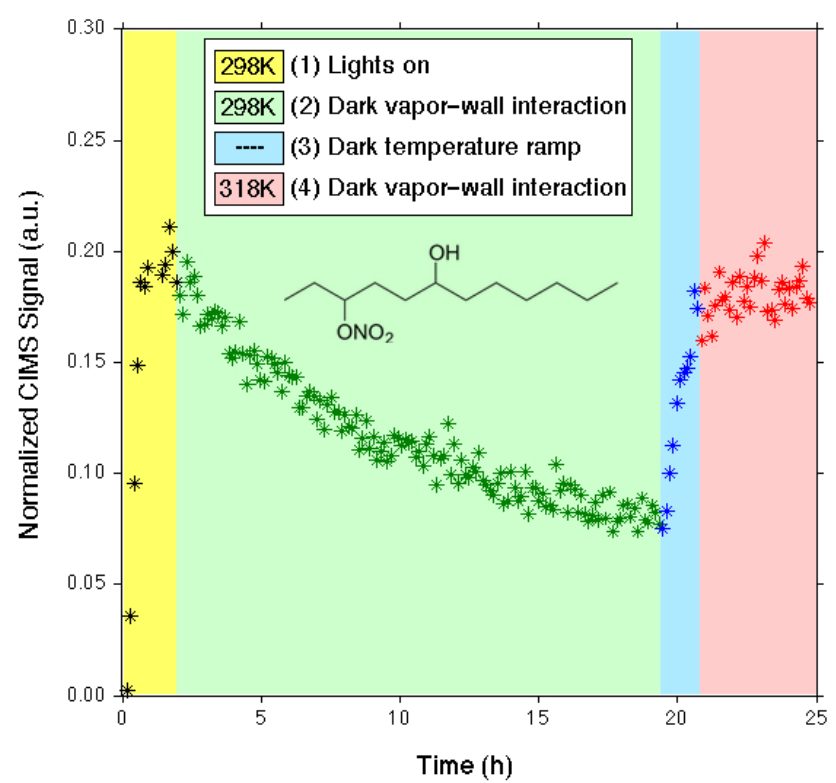

Figure 2. Example of the experimental procedure to assess vapor wall deposition using 3-nitrooxy-6-dodecanol $(\mathrm{m} / z=(-) 332)$ : period (1) organic oxidation product generation; period (2) vapor wall deposition at $298 \mathrm{~K}$ in the dark; period (3) chamber temperature ramp from 298 to $318 \mathrm{~K}$; and Period (4) temperature held at $318 \mathrm{~K}$ in the dark.

studies. Two control experiments were also conducted in two unused $24 \mathrm{~m}^{3}$ Teflon chambers using identical experimental protocols, see Table 1 .

Vapor molecules representing SOA products were generated directly via VOC photooxidation, as opposed to the external injection of commercially available chemical standards. In this manner, uncertainty in the initial vapor concentration due to filling and mixing is avoided. In order to generate a spectrum of oxidized compounds characterized by a combination of different carbon numbers and types of functional groups, isoprene, toluene, $\alpha$-pinene, and dodecane were chosen as the parent VOCs. Prior to each experiment, the Teflon chambers were flushed with purified dry air for $12 \mathrm{~h}$ at $45^{\circ} \mathrm{C}$, then "conditioned" by UV irradiation for $24 \mathrm{~h}$ in the presence of $2 \mathrm{ppm} \mathrm{H}_{2} \mathrm{O}_{2}$, followed by purging with purified dry air for $\sim 4$ days at $25^{\circ} \mathrm{C}$. Experiments were carried out under conditions in which the peroxy radicals formed from the initial $\mathrm{OH}$ reaction with the parent hydrocarbon react either primarily with NO (so-called high-NO) or $\mathrm{HO}_{2}$ and $\mathrm{RO}_{2}$ (so-called low-NO). For low-NO conditions, hydrogen peroxide $\left(\mathrm{H}_{2} \mathrm{O}_{2}\right)$ was used as the $\mathrm{OH}$ source by evaporating $120 \mu \mathrm{L}$ of $50 \%$ wt aqueous solution into the chamber with $5 \mathrm{~L} \mathrm{~min}^{-1}$ of purified air for $\sim 110 \mathrm{~min}$, resulting in an approximate starting $\mathrm{H}_{2} \mathrm{O}_{2}$ mixing ratio of $2.0 \mathrm{ppm}$. For high-NO conditions, nitrous acid (HONO) was used as the $\mathrm{OH}$ source by dropwise addition of $15 \mathrm{~mL}$ of $1 \mathrm{wt} \%$ $\mathrm{NaNO}_{2}$ into $30 \mathrm{~mL}$ of $10 \mathrm{wt} \% \mathrm{H}_{2} \mathrm{SO}_{4}$ in a glass bulb and introduced into the chambers with $5 \mathrm{~L} \mathrm{~min}^{-1}$ of purified air 
Table 1. Experimental conditions for production of oxidized organic vapors.

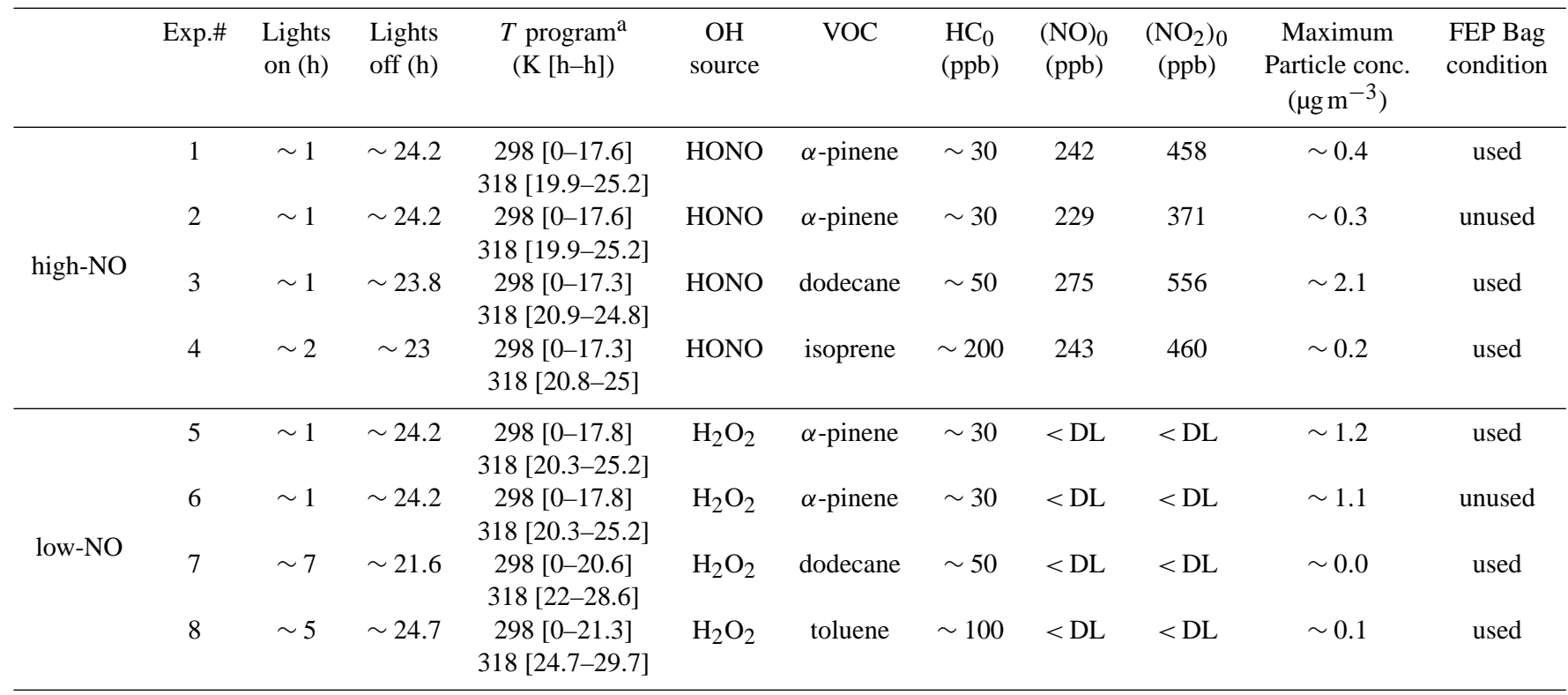

a The temperature is controlled at $298 \mathrm{~K}$ for the first $\sim 20 \mathrm{~h}$ of the experiment, including $\sim 1-7 \mathrm{~h}$ irradiation and $\sim 13-16 \mathrm{~h}$ darkness, and then ramped up to $318 \mathrm{~K}$ within $\sim 3 \mathrm{~h}$ and held for $\sim 4-6 \mathrm{~h}$

for $\sim 40 \mathrm{~min}$. Ozone formation is substantially limited in the presence of a high concentration of $\mathrm{HONO}$, and $\mathrm{NO}_{3}$ formation is negligible. A measured volume of hydrocarbon (isoprene/toluene/ $\alpha$-pinene/dodecane) was injected via a syringe into a glass bulb, which was connected to the Teflon chamber. Heated $5 \mathrm{Lmin}^{-1}$ of purified air flowed through the glass bulb into the chamber for $20 \mathrm{~min}$, introducing 25$200 \mathrm{ppb}$ of hydrocarbon into the chamber. After $\sim 60 \mathrm{~min}$ mixing, photooxidation was initiated by irradiating the chamber with black lights with output wavelength ranging from 300 to $400 \mathrm{~nm}$. Over the course of the irradiation period, the maximum particle mass concentration formed via nucleation ranged from 0.3 to $2 \mu \mathrm{g} \mathrm{m}^{-3}$, corresponding to a particle surface area to chamber wall area ratio of $<10^{-5}$. Under these conditions, the surface area of particles present in the chamber is sufficiently low that partitioning of organic vapors onto particles is negligible. After $\sim 1-7 \mathrm{~h}$ of reaction, UV lights were turned off and the decay of oxidation products due to wall deposition was monitored for $\sim 13-16 \mathrm{~h}$ at $25^{\circ} \mathrm{C}$. The chamber temperature was then ramped up to $45^{\circ} \mathrm{C}$ during the remaining $\sim 4-6 \mathrm{~h}$ of the experiment with other conditions held constant.

Gas-phase organic compounds were monitored using a custom-modified Varian 1200 triple-quadrupole CIMS (Crounse et al., 2006; Paulot et al., 2009). In negative-mode operation, $\mathrm{CF}_{3} \mathrm{O}^{-}$was used as the reagent ion to cluster with analytes [R] with strong fluorine affinity such as hydroperoxide, producing $\left[\mathrm{R} \cdot \mathrm{CF}_{3} \mathrm{O}\right]^{-}$or $m / z=[M+85]^{-}$, where $M$ is the molecular weight of the analyte. For more strongly acidic species $[\mathrm{X}]$, the transfer product, $\left[\mathrm{X}_{[\mathrm{H}]} \cdot \mathrm{HF}\right]^{-}$ or $m / z=[M+19]^{-}$, is formed during ionization. Carboxylic acids tend to have contributions to both the transfer and cluster products, in which case the trace with higher signal-tonoise ratio is considered. Prior to each experiment, the purified air in the chamber was sampled, and this is subtracted off as the CIMS background signal. The background signal is fairly consistent between the masses and over time. However, this background subtraction does not guarantee that the background for every $m / z$ signal is absolutely zero, as noted in Fig. 3 that the CIMS background for certain ions is hovering around zero. Identification of products by CIMS from the photooxidation of isoprene, $\alpha$-pinene, and dodecane in our laboratory has been previously reported (Paulot et al., 2009; Eddingsaas et al., 2012; Yee et al., 2012; Zhang et al., 2014b).

\section{Absorbing organic mass on the chamber wall $\left(C_{\mathrm{w}}\right)$}

Figure 3 shows the continuous dark decay of the 25 organic vapors generated from the photooxidation of isoprene, toluene, $\alpha$-pinene, and dodecane under high/low-NO conditions. In contrast to the behavior in Fig. 3, Matsunaga and Ziemann (2010) and Yeh and Ziemann (2014) observed rapid equilibrium established within less than an hour for vapor wall losses of $n$-alkanes, 1-alkenes, 2-alcohols, 2-ketones, monoacids, and 1,2-diols in both 1.7 and $5.9 \mathrm{~m}^{3}$ Teflon chambers. The organic vapor generation period in the present study ranges from 1 to $7 \mathrm{~h}$, thus precluding the possibility of observing more rapid partitioning that may have occurred. In view of this, we carried out one vapor wall deposition experiment in the $\alpha$-pinene $+\mathrm{OH}$ low-NO system, with the 

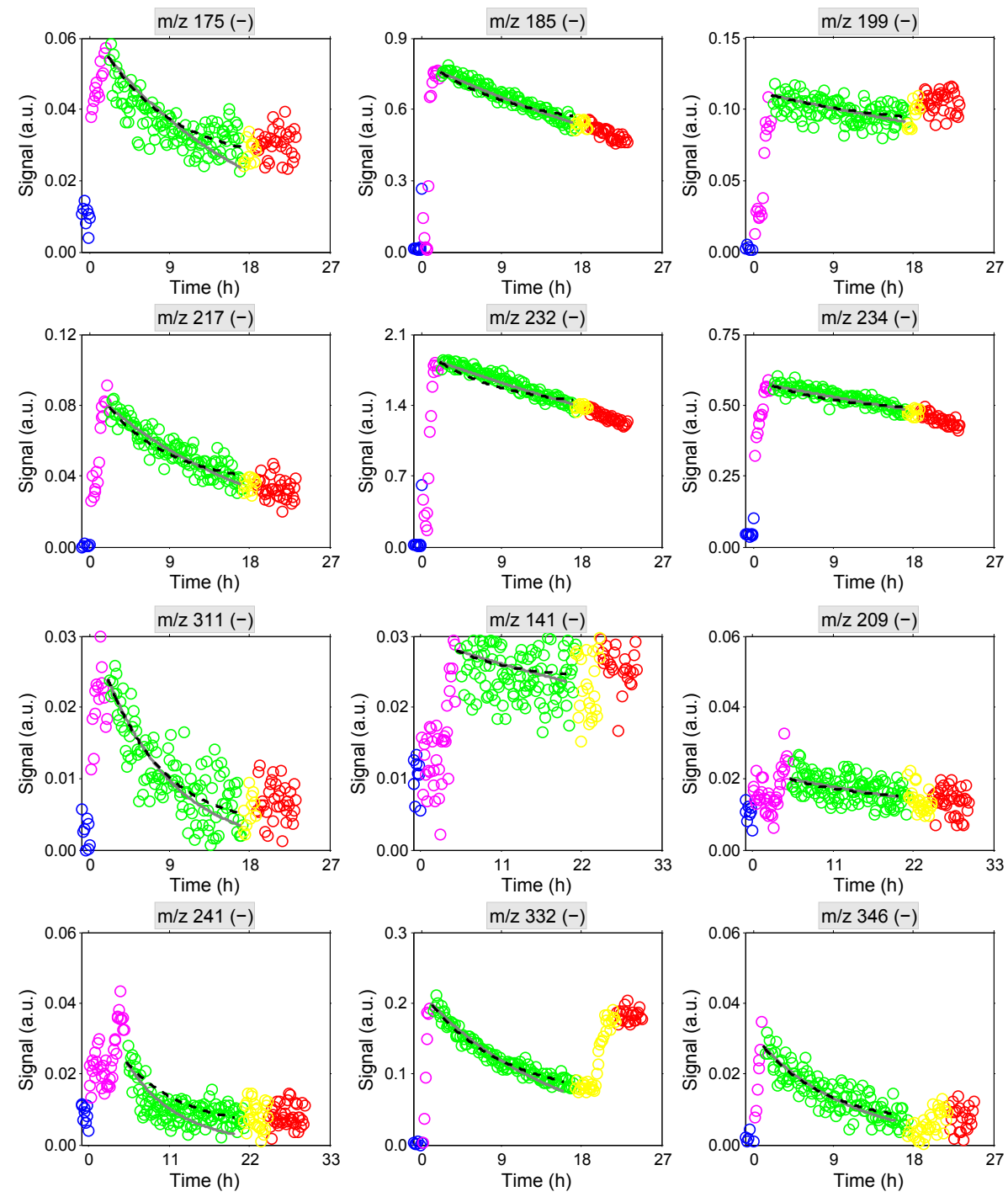

Figure 3.

experimental procedures identical to those in Sect. 3, but with lights on for only $10 \mathrm{~min}$. We also increased the initial mixing ratios of $\alpha$-pinene and $\mathrm{OH}$ radical precursor $\mathrm{H}_{2} \mathrm{O}_{2}$ to 1 and $4 \mathrm{ppm}$, respectively, in order to generate sufficient organic vapor CIMS signals during the short irradiation period. Prompt formation of two ions, $m / z 269(-)$ and $m / z 285(-)$, was observed on the CIMS after 10 min of photochemistry. These are assigned to be two first-generation products, pinonic acid $\left(\mathrm{C}_{10} \mathrm{H}_{16} \mathrm{O}_{3}\right)$ and pinonic peroxy acid $\left(\mathrm{C}_{10} \mathrm{H}_{16} \mathrm{O}_{4}\right)$, respectively (see Table 2 for the proposed chemical structures). Owing to the short photochemical reaction timescale, the other four possible products in Table 2 were not found in this experiment. Figure 3 (bottom panel) shows the wall induced dark decay of $m / z 269(-)$ and $m / z 285(-)$ at $298 \mathrm{~K}$. The best-fit first-order decay rates lie within the same order of magnitude as those reported in Table 2, i.e., $7.61 \times 10^{-6} \mathrm{~s}^{-1}$ vs. $8.95 \times 10^{-6} \mathrm{~s}^{-1}$ for $\mathrm{m} / z 269(-)$ and $1.67 \times 10^{-6} \mathrm{~s}^{-1}$ vs. $2.98 \times 10^{-6} \mathrm{~s}^{-1}$ for $m / z, 285(-)$. No rapid vapor wall loss was found immediately after lights off, and the deposition rates for both ions were pretty consistent over the course of $\sim 15 \mathrm{~h}$ dark decay. Note that $m / z 285$ (-), although having a higher molecular weight, decays more slowly than $m / z 269$ $(-)$. We will demonstrate later that the wall-induced decay rate depends inversely on the vapor pressure, which is a function of the molecule size and functionalities. The addition of a carboxylic acid group, as in $m / z 269$ (-), leads to a greater decrease in volatility than that resulting from the addition of a peroxy carboxylic acid group, as in $m / z 285$ (-). Our observations for these two compounds are consistent with the observed behavior of the other 23 compounds. There are three considerations regarding equipment setup and experimental protocol that potentially contribute to the differences between the present study and Ziemann and co-worker's work: (1) chamber size and depletion rate; (2) mixing status, i.e., 

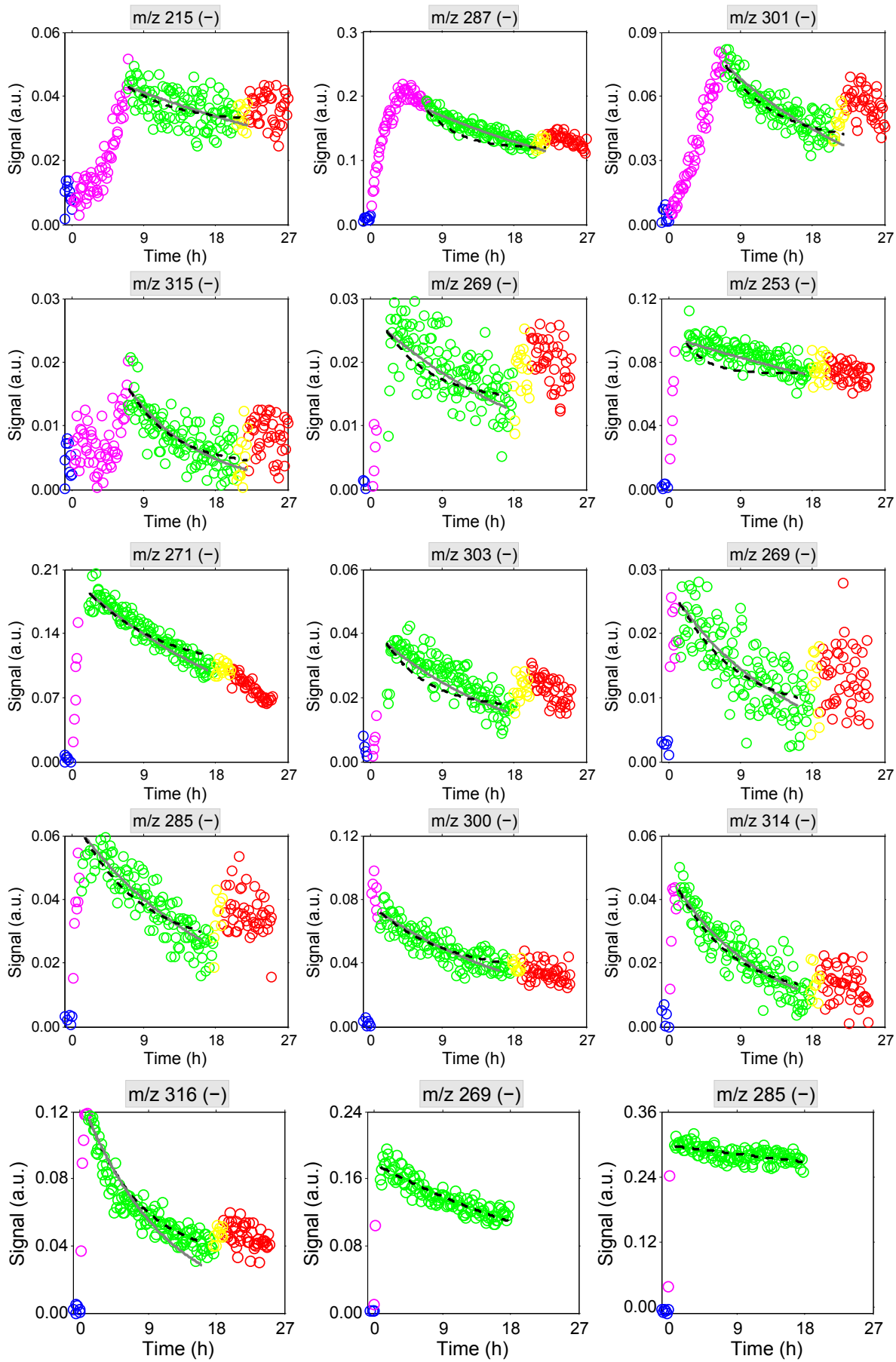

○ Background

- Lights on

- Dark vapor \pm wall interaFtion at $298 \mathrm{~K}$

○ Dark vapor \pm wall interaFtion at $318 \mathrm{~K}$

Dark temperature ramp

- - - SIM.1

SIM.2

Figure 3. CIMS traces of oxidized organic vapors generated from the photooxidation of isoprene, toluene, $\alpha$-pinene and dodecane under high/low-NO conditions over the four chamber periods in Fig. 2. Colored circles represent CIMS measured normalized signals during background (blue), vapor generation (magenta), vapor wall deposition at $298 \mathrm{~K}$ (green), temperature ramp (yellow), and vapor re-evaporation at $318 \mathrm{~K}$ (red). Black dashed lines and gray solid lines represent the simulated deposition rates generated from SIM.1 and SIM.2, respectively. 

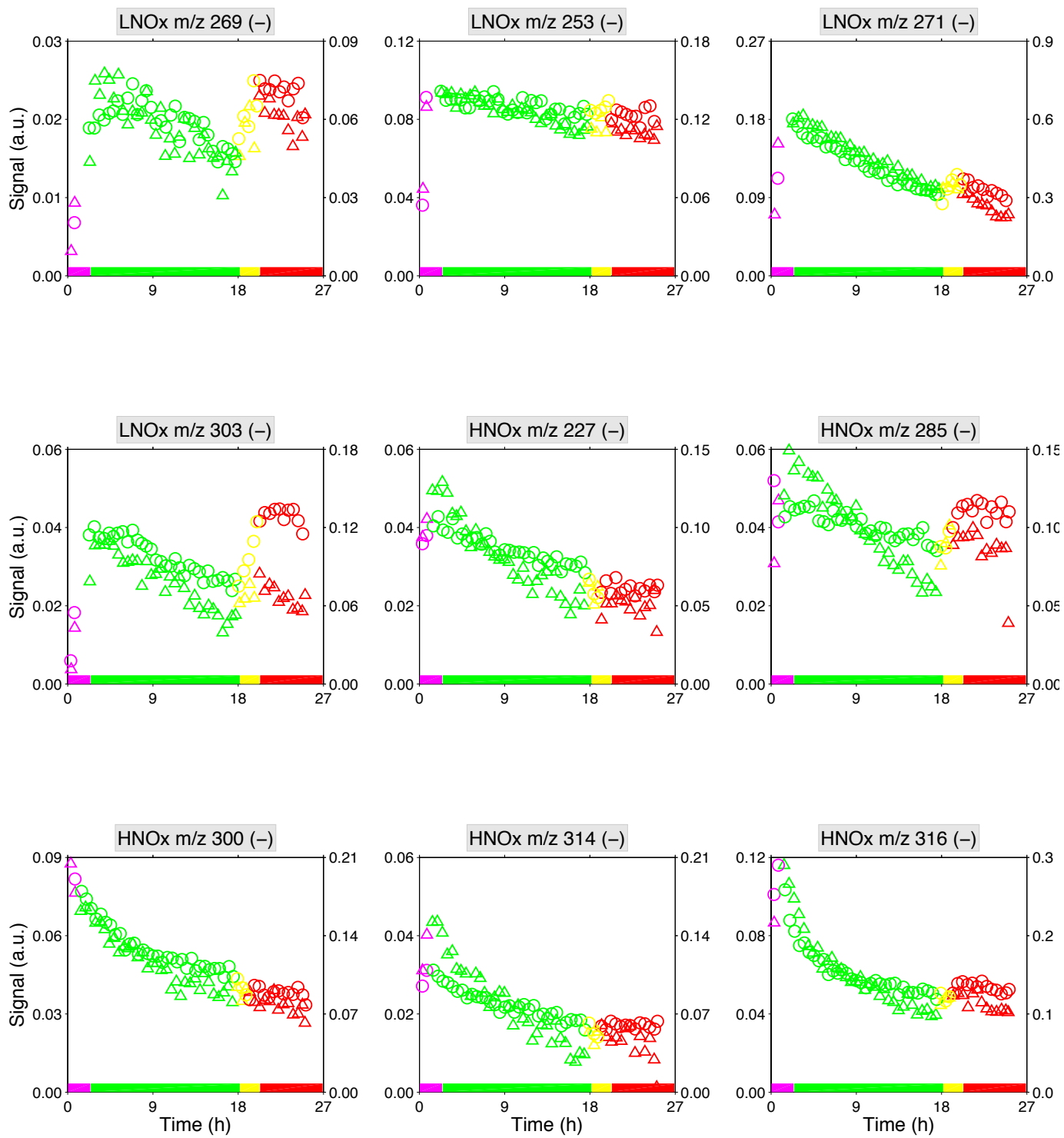

Figure 4. Comparison of vapor-wall interactions for $\alpha$-pinene $+\mathrm{OH}$ products under controlled experimental conditions in used (triangle) vs. unused (circle) Teflon chambers. 30-min averaged data are shown here for clarity. Colored bands denote successive experimental periods: vapor generation (magenta), vapor wall deposition at $298 \mathrm{~K}$ (green), temperature ramp (yellow), and vapor re-evaporation at $318 \mathrm{~K}$ (red).

actively mixed vs. static; and (3) definition of the starting point of the gas-phase vapor concentration.

When the chamber temperature was increased from 25 to $45^{\circ} \mathrm{C}$, with all the other experimental conditions held constant, the concentrations of most compounds in the chamber increased to a minor degree relative to the initial peak signal, reflecting modest desorption of vapors from the chamber wall. As noted earlier, the chamber wall (in the used chambers) might actually be coated with organic materials from previous experiments, or the FEP Teflon film itself may act as an absorbing medium. In view of the uncertain nature of the wall itself, two control experiments were also conducted in the unused dual $24 \mathrm{~m}^{3}$ FEP Teflon chambers with identical protocols: see Table 1. Organic vapor deposition and evapo- ration rates between unused and used chambers are compared in Fig. 4. For all the $\alpha$-pinene photooxidation products studied here, their interaction with the wall in the unused chambers is in general agreement with that in the used chambers, except for a few oxidation products generated under high-NO conditions. The fact that these particular compounds exhibit slightly higher deposition rates in used chambers might be due to the heterogeneous chemistry on the wall catalyzed by nitric acid, a product from the $\mathrm{NO}_{x}-\mathrm{O}_{3}$ photochemical cycle. Overall, we conclude that the extent to which chambers have been previously used is not a significant factor in the sorption behavior of the FEP Teflon films.

The equivalent absorbing organic mass parameter $\left(C_{\mathrm{w}} / \mathrm{g} \mathrm{m}^{-3}\right)$ is estimated using equilibrium partitioning 
Table 2. Best-fit values of vapor-wall accommodation coefficient $\left(\alpha_{\mathrm{w}, i}\right)$ and calculated equivalent absorbing organic mass $\left(C_{\mathrm{W}}\right)$ on the chamber wall for vapors with structure proposed based on the CIMS measurement.

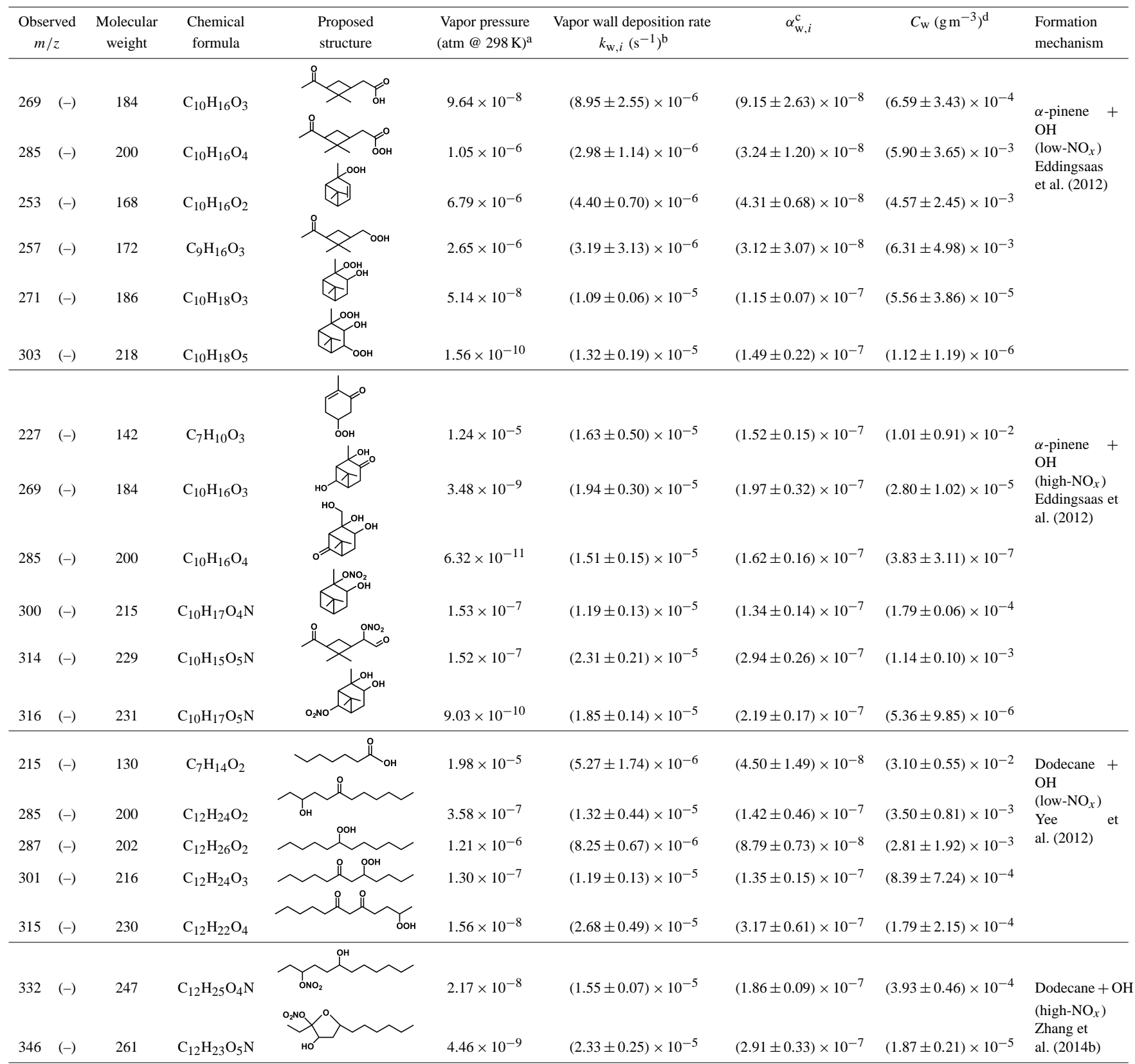


Table 2. Continued.

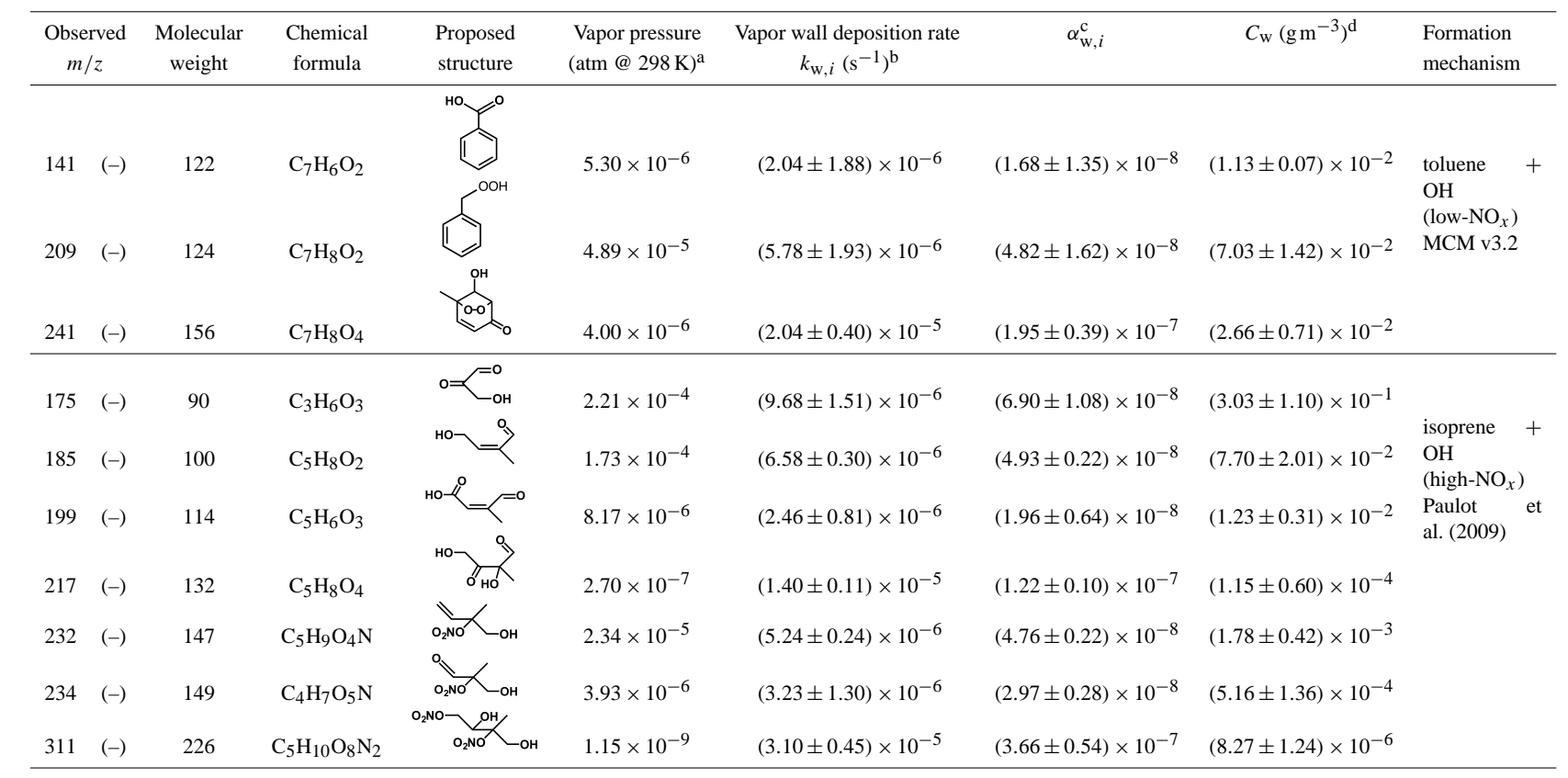

a Vapor pressures are estimated from the average of predictions from the two group contribution methods, "SIMPOL.1" (Pankow and Asher, 2008) and "EVAPORATION" (Compernolle et al., 2011).

$\mathrm{b}$ The vapor wall deposition rate $\left(k_{\mathrm{w}, i}\right)$ is calculated by Eq. (22b).

$\mathrm{c}$ The accommodation coefficient $\left(\alpha_{\mathrm{w}, i}\right)$ is calculated via optimal fitting of Eq. (22b) to the CIMS measured vapor decay rate assuming first-order kinetics and irreversible gas-wall partitioning.

${ }^{\mathrm{d}} C_{w}$ is calculated from the combination of Eqs. (16) and (17) as an equation set.
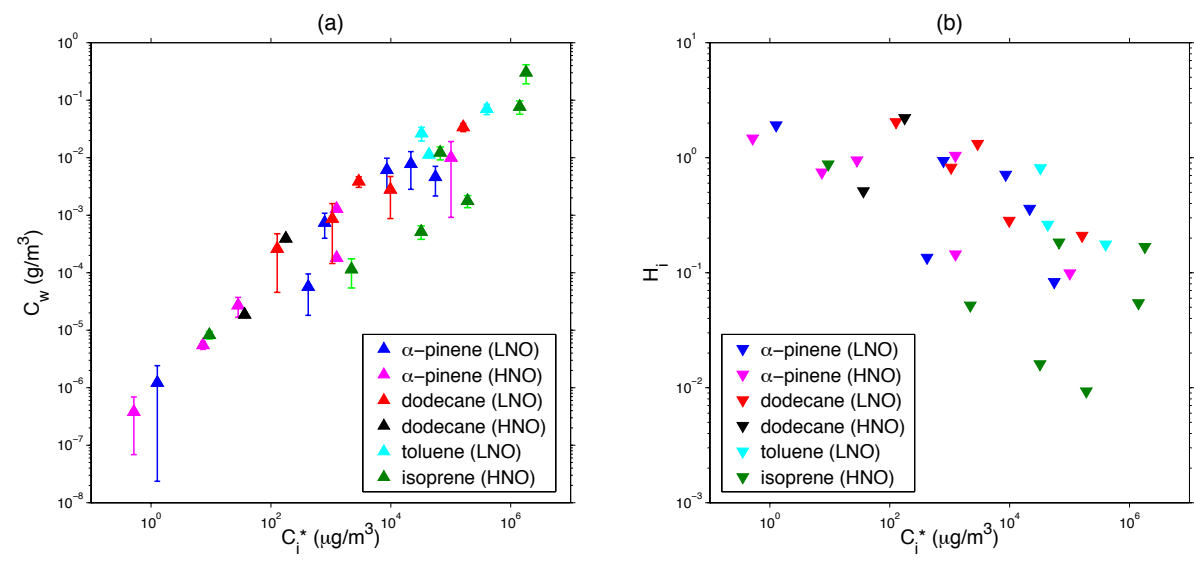

Figure 5. Inferred total amount of (a) equivalent absorbing organic mass on the chamber wall, $C_{\mathrm{w}}\left(\mathrm{g} \mathrm{m}^{-3}\right)$, and (b) dimensionless Henry's law constants, $H_{i}$, as a function of saturation concentration, $C_{i}^{*}\left(\mu \mathrm{g} \mathrm{m}^{-3}\right)$. Estimated vapor pressures of organic compounds studied here are obtained from the average of predictions from the two group contribution methods, "SIMPOL.1" (Pankow and Asher, 2008) and "EVAPORATION" (Compernolle et al., 2011). The uncertainty bars give the upper and lower limits of $C_{\mathrm{W}}$ values derived from Eq. (12), together with Eqs. (16) and (17), when either "EVAPORATION" or "SIMPOL.1" is used to estimate vapor pressures.

theory. We show in the Supplementary Materials that this theory is suitable for $C_{\mathrm{w}}$ estimation after $\sim 18 \mathrm{~h}$ of wallinduced vapor decay. The ratio of the concentration of vapor $i$ in the wall phase $\left(\bar{C}_{\mathrm{w}, i}\right)$ to that in the gas phase $\left(\bar{C}_{\mathrm{v}, i}\right)$ is expressed as a function of the corresponding gas-wall partitioning coefficient $\left(K_{\mathrm{w}, i}\right)$ and the total amount of equivalent absorbing organic mass on the chamber wall $\left(C_{\mathrm{w}}\right)$. Ideally,
$C_{\mathrm{w}}$ can be obtained if the initial total concentration $\left(\bar{C}_{\mathrm{tot}, i}\right)$ and equilibrium gas-phase concentration $\left(\bar{C}_{\mathrm{v}, i}\right)$ of vapor $i$ can be measured by CIMS. However, since the fraction of organic compound $i$ in the chamber wall at the onset of vapor wall deposition is unknown, we estimate $C_{\mathrm{w}}$ via the combination of equilibrium partitioning expressions at two 
different temperatures, e.g., 298 and $318 \mathrm{~K}$ :

$$
\begin{aligned}
& \frac{\bar{C}_{\mathrm{w}, i @ 298 \mathrm{~K}}}{\bar{C}_{\mathrm{v}, i @ 298 \mathrm{~K}}}=\frac{\bar{C}_{\mathrm{tot}, i}-\bar{C}_{\mathrm{v}, i @ 298 \mathrm{~K}}}{\bar{C}_{\mathrm{v}, i @ 298 \mathrm{~K}}}=K_{\mathrm{w}, i @ 298 \mathrm{~K}} C_{\mathrm{w}}, \\
& \frac{\bar{C}_{\mathrm{w}, i @ 318 \mathrm{~K}}}{\bar{C}_{\mathrm{v}, i @ 318 \mathrm{~K}}}=\frac{\bar{C}_{\mathrm{tot}, i}-\bar{C}_{\mathrm{v}, i @ 318 \mathrm{~K}}}{\bar{C}_{\mathrm{v}, i @ 318 \mathrm{~K}}}=K_{\mathrm{w}, i @ 318 \mathrm{~K}} C_{\mathrm{w}},
\end{aligned}
$$

where $\bar{C}_{\text {tot }, i}$ is the total initial concentration of vapor $i$, $\bar{C}_{\mathrm{v}, i @ 298 / 318 \mathrm{~K}}$ is the gas-phase concentration (as indicated by the normalized CIMS signal with unit "a.u.") of vapor $i$ at $298 / 318 \mathrm{~K}$, and $K_{\mathrm{W}, i @ T}$ is the corresponding partitioning coefficient at temperature $T$, see Eq. (12). In this manner, both $\bar{C}_{\text {tot, } i}$ and $C_{\mathrm{w}}$ can be calculated by solving the equation set (16) and (17). Note that the product $K_{\mathrm{w}, i @ T} C_{\mathrm{w}}$ is dimensionless, so that the normalized CIMS signal can be directly substituted into Eqs. (16) and (17) as the actual gas-phase concentration of organic vapor $i$. In the calculation, $\bar{C}_{\mathrm{v}, i @ 298 \mathrm{~K}}$ and $\bar{C}_{\mathrm{v}, i @ 318 \mathrm{~K}}$ were obtained by taking a $30 \mathrm{~min}$ average of the first-order extrapolation of the normalized CIMS signals at 298 and $318 \mathrm{~K}$, respectively, during the temperature ramping period. The estimated $C_{\mathrm{w}}$ values vary by approximately 5 orders of magnitude and exhibit a strong dependence on the volatility of the organics, as shown in Table 2 and Fig. 5a. We will address subsequently why the $C_{\mathrm{w}}$ values span such a wide range.

\section{Vapor sorption into FEP Teflon films}

It is instructive to consider possible mechanisms of organic vapor interactions with Teflon films. Dual sorption mechanisms in glassy polymers have been identified: ordinary dissolution and microvoid-filling (Meares, 1954; Paul, 1979; Paterson et al., 1999; Tsujita, 2003; Kanehashi and Nagai, 2005). From the point of view of solubility behavior, organic polymers such as FEP Teflon may be idealized as high molecular weight organic liquids (Vieth et al., 1966). The polymer rubbery state is hypothesized to represent a thermodynamic equilibrium liquid state within which gas solubility obeys Henry's law. The glassy state, on the other hand, is considered to comprise two components: a hypothetical liquid state and a solid state, the latter containing a distribution of microvoids/holes that act to immobilize a portion of the penetrant molecules when the polymer is below its glass transition temperature $\left(T_{\mathrm{g}}=339 \mathrm{~K}\right.$ for FEP, Kim and Smith, 1990). The overall solubility of a gas molecule in a glassy polymer has been expressed by (Barrer et al., 1958; Michaels et al., 1963; Vieth et al., 1966; Kanehashi and Nagai, 2005):

$C=C_{\mathrm{H}}+C_{\mathrm{L}}=k_{\mathrm{H}} p+\frac{C_{\mathrm{L}}^{\prime} b p}{1+b p}$,

where $C$ is the total vapor concentration in the glassy polymer, $C_{\mathrm{H}}$ is the concentration based on Henry's law dissolution, $C_{\mathrm{L}}$ is the concentration based on Langmuir sorption, $k_{\mathrm{H}}$ is the Henry's law constant, $p$ is the partial pressure in the gas phase, $C_{\mathrm{L}}^{\prime}$ is the hole saturation constant, and $b$ is the hole affinity constant. If $b p \ll 1$, Eq. (18) reduces to

$C=\left(k_{\mathrm{H}}+C_{\mathrm{L}}^{\prime} b\right) p$.

The condition of $b p \ll 1$ holds in the present situation because the partial pressures of organic vapors generated in the chamber are $<10^{-7} \mathrm{~atm}$, and the derived hole affinity constants for small organic molecules are $<1 \mathrm{~atm}^{-1}$ in glassy polymers (Vieth et al., 1966; Sada et al., 1988; Kanehashi and Nagai, 2005). If Eq. (18) holds for the equilibrium sorption behavior of organic vapors by FEP films, then the dimensionless form of the effective Henry's law constant $\left(H_{i}\right)$ can be expressed as a function of the partitioning coefficient of vapor $i\left(K_{\mathrm{W}, i}\right)$ and total absorbing organic mass on the chamber wall $\left(C_{\mathrm{w}}\right)$ :

$H_{i}=\frac{\bar{C}_{\mathrm{w}, i}}{\bar{C}_{\mathrm{v}, i}}=K_{\mathrm{w}, i} C_{\mathrm{w}} \propto\left(k_{\mathrm{H}}+C_{\mathrm{L}}^{\prime} b\right)$.

As shown in Fig. 5b, the derived Henry's law constants $\left(H_{i}\right)$ for the organic oxidation products span approximately 2 orders of magnitude and depend inversely on saturation concentrations $\left(C_{i}^{*} / \mu \mathrm{g} \mathrm{m}^{-3}\right)$. This behavior suggests that organic vapor solubility in FEP films increases with decreasing volatility, i.e., increasing carbon number and functionalization. This behavior provides a qualitative explanation for the wide range of $C_{\mathrm{w}}$ values calculated for the 25 organic vapors studied here. Although the solubility of low volatility vapors in the FEP Teflon film is relatively high (large $H_{i}$ ), the total equivalent absorbing organic mass on the wall required for gas-wall partitioning can still be low (small $C_{\mathrm{w}}$ ) because low volatility compounds tend to partition preferentially in the wall phase (large $K_{\mathrm{w}, i}$ ). As illustrated in Fig. 5b, the dimensionless Henry's law constant of $m / z=(-) 303$, a product from $\alpha$-pinene low-NO photochemistry, is $\sim 20$ times larger than that of $m / z=(-) 185$, a product from isoprene $+\mathrm{OH}$ under high-NO conditions. The vapor pressure of $m / z=(-) 303$, however, is $\sim 6$ orders of magnitude lower than that of $m / z=(-) 185$. As a result, the $C_{\mathrm{w}}$ value for $m / z=(-) 303$ is $\sim 5$ orders of magnitude smaller than that for $m / z=(-) 185$. One infers that the equivalent absorbing organic mass on the chamber wall derived earlier is not constant but specific to individual organic compounds, i.e., a function of volatility and solubility in FEP Teflon polymer. We will show that $C_{\mathrm{w}}$ is not the most dominant parameter, so the assumption of a single value for $C_{\mathrm{w}}$, does not invalidate the usefulness of the theory.

\section{Accommodation coefficient on the chamber wall $\left(\alpha_{\mathrm{w}, i}\right)$}

One key parameter that emerges from the theory of vapor wall deposition, the total equivalent absorbing organic mass $\left(C_{\mathrm{w}}\right)$, can be calculated based on equilibrium gas-wall partitioning at two different temperatures. From this information, 
we can estimate the other key parameter, the accommodation coefficient $\left(\alpha_{\mathrm{w}, i}\right)$, by optimal fitting of the solution of Eq. (14) to CIMS measured organic vapor decay at $298 \mathrm{~K}$ :

$$
\begin{aligned}
\frac{d \bar{C}_{\mathrm{v}, i}}{\mathrm{~d} t} & =\left(\frac{A}{V}\right)\left(\frac{\alpha_{\mathrm{w}, i} \bar{v}_{i} / 4}{\pi \alpha_{\mathrm{w}, i} \bar{v}_{i} / 8\left(D_{i} K_{\mathrm{e}}\right)^{1 / 2}+1}\right) \\
& \cdot\left(\frac{\bar{C}_{\mathrm{tot}, i}-\bar{C}_{\mathrm{v}, i}}{K_{\mathrm{w}, i} C_{\mathrm{w}}}-\bar{C}_{\mathrm{v}, i}\right) .
\end{aligned}
$$

Note that Eq. (21) is simply Eq. (14) in which $\bar{C}_{\mathrm{w}, i}$ has been replaced with $\left(\bar{C}_{\text {tot }, i}-\bar{C}_{\mathrm{v}, i}\right)$. Thus, Eq. (21) constitutes a linear ODE system with the one unknown (estimable) parameter, $\alpha_{\mathrm{w}, i}$. The Levenberg-Marquardt method implemented in MATLAB's "System Identification Toolbox" was used for the nonlinear minimization at each time step of its solution. The best-fit $\alpha_{\mathrm{w}, i}$ value obtained was then substituted into Eq. (21) to give the simulated temporal profile of the organic vapor $i$. Simulation results (SIM.1) are shown in Fig. 3.

The other limit of wall behavior is that of irreversible gas-wall partitioning $\left(C_{\mathrm{w}} \rightarrow \infty\right)$. In this case, the accommodation coefficient $\alpha_{\mathrm{w}, i}$ is the sole governing parameter and Eq. (14) can be simplified as

$$
\frac{d \bar{C}_{\mathrm{v}, i}}{\mathrm{~d} t}=-\left(\frac{A}{V}\right)\left(\frac{\alpha_{\mathrm{w}, i} \bar{v}_{i} / 4}{\pi \alpha_{\mathrm{w}, i} \bar{v}_{i} / 8\left(D_{i} K_{\mathrm{e}}\right)^{1 / 2}+1}\right) \bar{C}_{\mathrm{v}, i} .
$$

The overall wall loss rate of organic vapor $i\left(k_{\mathrm{w}, i}\right)$ is therefore

$k_{\mathrm{w}, i}=\left(\frac{A}{V}\right)\left(\frac{\alpha_{\mathrm{w}, i} \bar{v}_{i} / 4}{\pi \alpha_{\mathrm{w}, i} \bar{v}_{i} / 8\left(D_{i} K_{\mathrm{e}}\right)^{1 / 2}+1}\right)$.

Results for irreversible gas-wall partitioning (SIM.2) are shown in Fig.. 3.

Simulations using both reversible (SIM.1) and irreversible (SIM.2) vapor wall deposition expressions match the experimental data. Outputs from SIM.1 tend to level off, whereas those from SIM.2 exhibit a continuous decreasing trend at the end of $\sim 18 \mathrm{~h}$ of vapor decay. The extent of agreement between observations and simulations depends on the nature of vapor wall deposition: most organic vapors in the Caltech Teflon chambers exhibit a continuous decay. The agreement between SIM.1 and SIM.2 indicates that the estimated $C_{\mathrm{w}}$ values are sufficiently large so that the wall-induced vapor deposition in the Caltech chamber can be treated as an irreversible process $\left(C_{\mathrm{w}} \rightarrow \infty\right)$ within a relatively long timescale $(<18 \mathrm{~h})$.

Overall, results from the two simulations indicate that $\alpha_{\mathrm{w}, i}$ is the more influential parameter than $C_{\mathrm{w}}$ in describing the wall-induced deposition of semi-volatile organic vapors. The significance of $\alpha_{\mathrm{w}, i}$ is 2-fold: first, the accommodation coefficient for the desorption of organic molecules from the gaswall interface equals that for the adsorption/uptake process, which together influence the time needed to establish equilibrium; and second, diffusion in the chamber wall is not considered in the theoretical framework; consequently, the bestfit $\alpha_{\mathrm{w}, i}$ will reflect the mass transfer resistance in both the

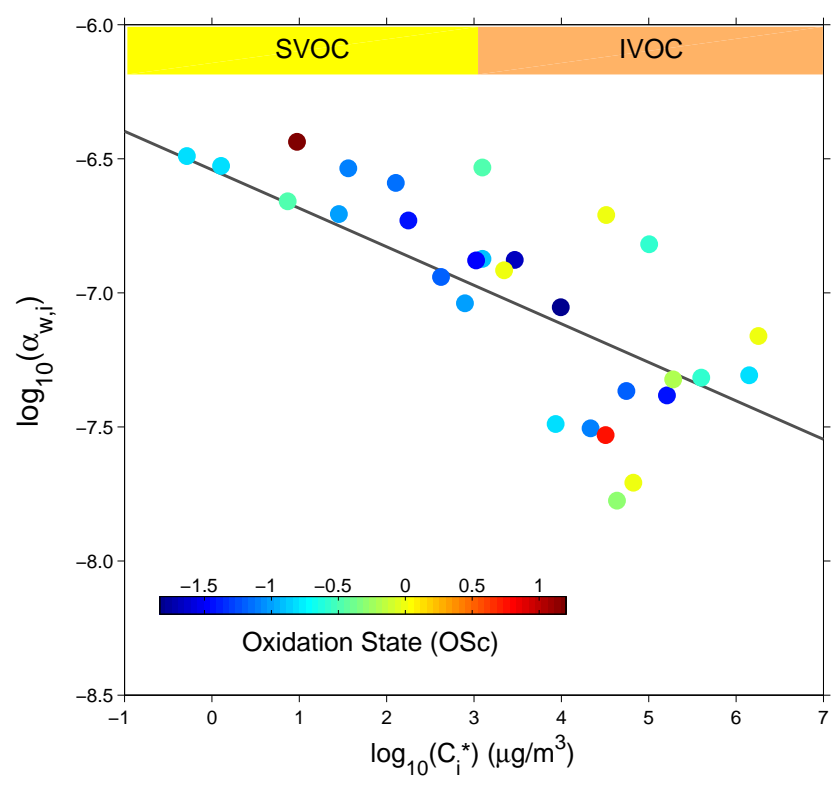

Figure 6. Inferred accommodation coefficients of organic oxidation products on the chamber wall $\left(\log _{10}\left(\alpha_{\mathrm{w}, i}\right)\right)$ as a function of saturation concentrations $\left(\log _{10}\left(C_{i}^{*}\right)\right)$ and average carbon oxidation state $\left(\mathrm{OS}_{\mathrm{C}}\right)$. Colored filled circles represent the best-fit $\alpha_{\mathrm{w}, i}$ assuming irreversible gas-wall partitioning. The black solid line represents the linear regression of $\log _{10}\left(\alpha_{\mathrm{w}, i}\right)$ vs. $\log _{10}\left(C_{i}^{*}\right)$ for all compounds.

gas-wall interface and the chamber wall layer. We suggest that the vapor wall deposition of individual compounds can be adequately parameterized through the accommodation coefficient $\alpha_{\mathrm{w}, i}$ as the single dominant variable. As shown in Table 2 and Fig. 6, for the compounds studied here, estimated values of $\alpha_{\mathrm{w}, i}$ span approximately 2 orders of magnitude $\left(10^{-8}-10^{-6}\right)$ and depend inversely on volatility, implying that more highly functionalized compounds dissolve more easily in FEP Teflon film. The correlation of $\alpha_{\mathrm{w}, i}$ with the average carbon oxidation state $\left(\mathrm{OS}_{\mathrm{C}}\right)$, however, is not strong due to the fact that vapor pressures of molecules, although highly oxidized, are not necessarily low.

\section{Characterizing chamber vapor wall deposition rate}

The wall-induced deposition of the 25 organic compounds investigated in the present study can be sufficiently represented by a single parameter, the wall accommodation coefficient $\left(\alpha_{\mathrm{w}, i}\right)$, which is observed to exhibit a strong inverse dependence on $C_{i}^{*}$ (Fig. 6). It is possible to formulate an empirical expression for $\alpha_{\mathrm{w}, i}$ as a function of $C_{i}^{*}$, a parameter that can be estimated by vapor pressure prediction models.

Linear regression was performed on $\log _{10} \alpha_{\mathrm{w}, i}$ vs. $\log _{10} C_{i}^{*}$ for the 25 organic vapors studied:

$\log _{10} \alpha_{\mathrm{w}, i}=-0.1919 \times \log _{10} C_{i}^{*}-6.32$. 
We employ a group-contribution expression for $\log _{10} C_{i}^{*}$ as a function of carbon number $\left(n_{\mathrm{C}}^{i}\right)$ and oxygen number $\left(n_{\mathrm{O}}^{i}\right)$ developed by Donahue et al. (2011):

$\log _{10} C_{i}^{*}=\left(n_{\mathrm{C}}^{0}-n_{\mathrm{C}}^{i}\right) b_{\mathrm{C}}-n_{\mathrm{O}}^{i} b_{\mathrm{O}}-2 \frac{n_{\mathrm{C}}^{i} n_{\mathrm{O}}^{i}}{n_{\mathrm{C}}^{i}+n_{\mathrm{O}}^{i}} b_{\mathrm{CO}}$,

where $n_{\mathrm{C}}^{0}$ is the carbon number of $1 \mu \mathrm{g} \mathrm{m}{ }^{-3}$ alkane $\left(n_{\mathrm{C}}^{0}=\right.$ 28.0483), $b_{\mathrm{C}}$ is the carbon-carbon interaction term $\left(b_{\mathrm{C}}=\right.$ $0.4015), b_{\mathrm{O}}$ is the oxygen-oxygen interaction term $\left(b_{\mathrm{O}}=\right.$ $2.3335)$, and $b_{\mathrm{CO}}$ is the carbon-oxygen nonideality term $\left(b_{\mathrm{CO}}=-0.4709\right)$. Best-fit values of $n_{\mathrm{C}}^{0}, b_{\mathrm{C}}, b_{\mathrm{O}}$, and $b_{\mathrm{CO}}$ are obtained by optimal fitting Eq. (24) to the saturation concentrations of 110 species, including $\mathrm{C}_{5}-\mathrm{C}_{14} n$-alkanes, $\mathrm{C}_{5}-\mathrm{C}_{14}$ carbonyls, $\mathrm{C}_{5}-\mathrm{C}_{14}$ di-carbonyls, $\mathrm{C}_{5}-\mathrm{C}_{14}$ alcohols, $\mathrm{C}_{5}-\mathrm{C}_{14}$ diols, $\mathrm{C}_{5}-\mathrm{C}_{14}$ carboxylic acids, $\mathrm{C}_{5}-\mathrm{C}_{14}$ di-carboxylic acids, $\mathrm{C}_{5}-\mathrm{C}_{14}$ peroxides, $\mathrm{C}_{5}-\mathrm{C}_{14}$ di-peroxides, $\mathrm{C}_{5}-\mathrm{C}_{14}$ nitrates, and $\mathrm{C}_{5}-\mathrm{C}_{14}$ di-nitrates. Vapor pressures of these species are estimated by taking the average of predictions from the two group contribution methods, "SIMPOL.1" and "EVAPORATION".

Combining Eqs. (22), (23), and (24), the vapor wall deposition rate of any intermediate/semi/low-volatility compound $\left(k_{\mathrm{w}, i} / \mathrm{s}^{-1}\right)$ can be ultimately related to its carbon and oxygen numbers. This vapor wall loss rate estimation approach, although simplified, proves to be quite useful considering the limited knowledge of the chemical structures of the thousands of ions detected by mass spectrometry during an experiment. The proper guess of a molecular formula would be able to constrain the wall-induced decay rate of each ion, and thus provide information to better understand its formation and removal dynamics. In this way, one can reasonably constrain the wall-induced organic vapor deposition rate based on only two measurable or predictable properties, volatility and the extent of oxygenation.

As shown in Fig. 7, within a certain volatility range, $k_{\mathrm{w}, i}$ increases with decreasing $C_{i}^{*}$, implying that highly functionalized compounds tend to deposit on the chamber wall more efficiently. The maximum value of vapor wall deposition rate is eventually approached for highly oxygenated and extremely low-volatility compounds (which, of course, are precisely those compounds that are most prone to form SOA). Revisiting Eq. (22) reveals that the deposition rate of organic vapors is limited either by gas phase transport (molecular diffusion and turbulent mixing) or wall surface accommodation. For extremely small $\alpha_{\mathrm{w}, i}\left(\operatorname{large} C_{i}^{*}\right), k_{\mathrm{w}, i}$ becomes

$k_{\mathrm{w}, i}=\left(\frac{A}{V}\right)\left(\frac{\alpha_{\mathrm{w}, i} \bar{v}_{i}}{4}\right)$.

In this case, the organic vapor wall deposition rate is governed by the chamber wall accommodation process. On the other hand, if $\alpha_{\mathrm{w}, i}$ is sufficiently large (small $\left.C_{i}^{*}\right), k_{\mathrm{w}, i}$ is approximately given by

$k_{\mathrm{w}, i}=\frac{\pi}{2}\left(\frac{A}{V}\right)\left(D_{i} K_{\mathrm{e}}\right)^{1 / 2}$.

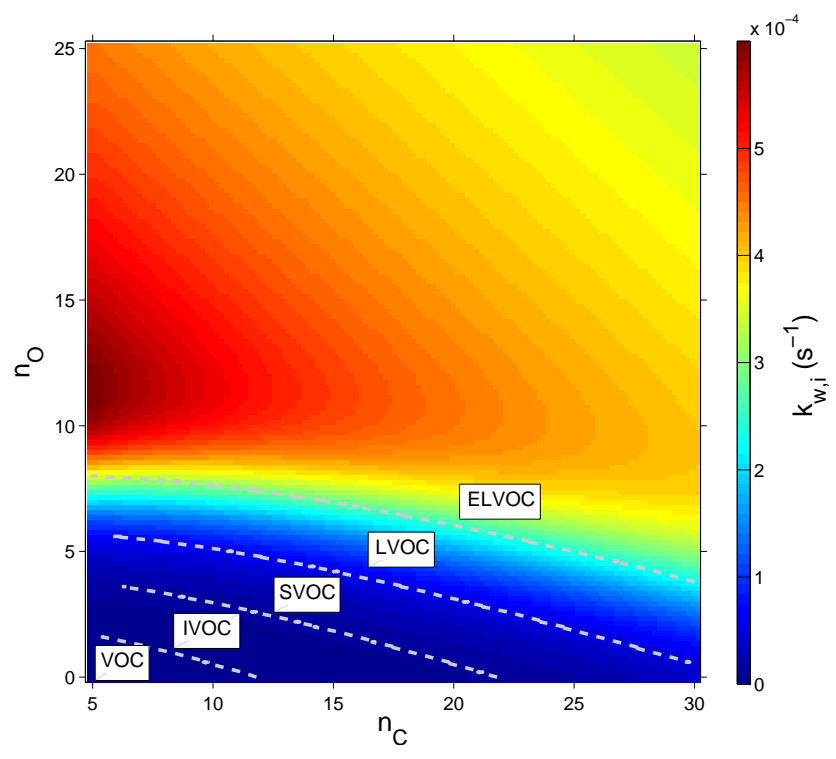

Figure 7. Predicted vapor wall deposition rate $\left(k_{\mathrm{w}, i} / \mathrm{s}^{-1}\right)$ of organic compounds in a Teflon chamber as a function of carbon number $\left(n_{\mathrm{C}}\right)$ and oxygen number $\left(n_{\mathrm{O}}\right)$.

In this case, the vapor wall deposition rate is ultimately controlled by the mixing state in the chamber. Equation (26) provides an expression for the upper limit of vapor wall deposition rate in a chamber, which is a manifestation of the extent of turbulent mixing in the chamber. One can determine which process is the limiting step in governing the overall wall deposition rate by referring to Eqs. (25) and (26). The threshold value of $\alpha_{\mathrm{w}, i}$, at which gas phase transport (molecular diffusion and turbulence mixing) and wall surface accommodation contribute equally to the vapor wall deposition rate, is $6.8 \times 10^{-6}$ in the Caltech chamber.

\section{Impact of vapor wall deposition on SOA yields}

The extent to which vapor wall deposition impacts measured SOA yields depends on the competition between uptake of organic vapors by suspended particles and the chamber wall. The timescale $\left(\tau_{\mathrm{g} / \mathrm{p}, i}\right)$ associated with establishing equilibrium gas-particle partitioning is governed by three transport processes: diffusion of vapor molecules from the bulk gas phase to the surface of the particle, uptake of vapor molecules by the particle surface, and diffusion of molecules in the bulk particle phase. Depending on a given situation, any of these three transport processes can be the limiting step in determining the overall equilibrium partitioning timescale. Here we represent the diffusional transport processes across the gasparticle interface and in the particle phase itself by a single parameter, the accommodation coefficient of organic vapors on the particle $\left(\alpha_{\mathrm{p}, i}\right)$. In doing so, the mass transfer resistances at the gas-particle interface and in the particle phase are reflected by the single parameter $\alpha_{\mathrm{p}, i}$, and the timescale 


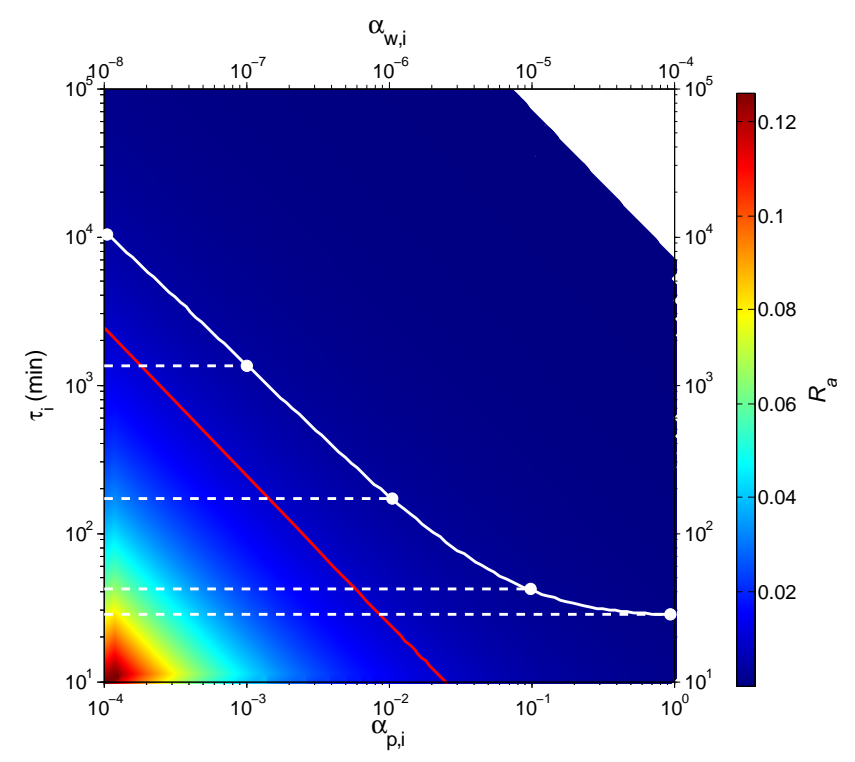

Figure 8. Comparison of estimated gas-particle equilibration timescale $\left(\tau_{\mathrm{g} / \mathrm{p}, i}\right)$ as a function of the gas-particle mass accommodation coefficient $\left(\alpha_{\mathrm{p}, i}\right.$, lower $x$ axis) and the ratio of total particle surface area to the chamber wall area $\left(R_{\mathrm{a}}\right.$, color bar), and vapor wall deposition timescale $\left(\tau_{\mathrm{g} / \mathrm{w}, i}\right)$ as a function of gas-wall mass accommodation coefficient $\left(\alpha_{\mathrm{w}, i}\right.$, upper $x$ axis). The red solid line represents the gas-particle equilibration time for a typical chamber experiment with seed surface area of $\sim 1 \times 10^{-3} \mu \mathrm{m}^{2} \mathrm{~cm}^{-3}$. White solid and dashed lines define the region where $\tau_{\mathrm{g} / \mathrm{p}, i} \cong \tau_{\mathrm{g} / \mathrm{w}, i}$. For example, the top dashed white line is a collection of data points for which the equality $\tau_{\mathrm{g} / \mathrm{p}, i}=\tau_{\mathrm{g} / \mathrm{w}, i}=1.3 \times 10^{3} \min$ holds. $\tau_{\mathrm{g} / \mathrm{w}, i}$ is calculated by substituting $\alpha_{\mathrm{W}, i}=10^{-7}$ into Eqs. (22), (23), and (24). $\tau_{\mathrm{g} / \mathrm{p}, i}$ is calculated from Eq. (27) by varying $\alpha_{\mathrm{p}, i}\left(10^{-4}-10^{-3}\right)$ and $R_{\mathrm{a}}(0.01-0.02)$.

to achieve gas-particle partitioning following a small perturbation of the condensing species in the gas phase is given by (Seinfeld and Pandis, 2006):

$\tau_{\mathrm{g} / \mathrm{p}, i}=\left(2 \pi N_{\mathrm{p}} \bar{D}_{\mathrm{p}} D_{i} f\left(K n, \alpha_{\mathrm{p}, i}\right)\right)^{-1}$,

where $N_{\mathrm{p}}$ is the total number concentration of suspended particles, $\bar{D}_{\mathrm{p}}$ is the number mean particle diameter, $K n(=$ $\left.2 \lambda / D_{\mathrm{p}}\right)$ is the Knudsen number, and $f\left(K n, \alpha_{\mathrm{p}, i}\right)$ is the correction factor for noncontinuum diffusion and imperfect accommodation (Seinfeld and Pandis, 2006).

Figure 8 shows the predicted $\tau_{\mathrm{g} / \mathrm{p}, i}$ as a function of: (1) the ratio of total particle surface area to chamber wall area $\left(R_{\mathrm{a}}\right)$ and (2) $\alpha_{\mathrm{p}, i}$. The red solid line represents $\tau_{\mathrm{g} / \mathrm{p}, i}$ for a typical chamber experiment with seed surface area of $\sim 1000 \mu \mathrm{m}^{2} \mathrm{~cm}^{-3}$. In this case, equilibrium vapor-particle partitioning is established within a few minutes in the presence of perfect accommodation of organic vapors onto particles $\left(\alpha_{\mathrm{p}, i}=1\right)$ or when a sufficiently large concentration of suspended particles is present (e.g., $C_{\mathrm{OA}}>10^{5} \mu \mathrm{g} \mathrm{m}^{-3}$ when $\left.\alpha_{\mathrm{p}, i}<10^{-4}\right)$.
By analogy with the treatment of gas-particle partitioning, the time scale associated with vapor-wall interactions is presumably governed by gas-phase diffusion of vapor molecules to the wall through a boundary layer adjacent to the wall, uptake of vapor molecules at the wall surface, and, potentially, diffusion of molecules in the wall. Again, a single parameter, the accommodation coefficient on the wall $\left(\alpha_{\mathrm{w}, i}\right)$, is employed to represent the latter two processes. Thus, the vapor wall deposition timescale is given by

$\tau_{\mathrm{g} / \mathrm{w}, i}=k_{\mathrm{w}, i}^{-1}$.

The white solid line in Fig. 8 represents the predicted $\tau_{\mathrm{g} / \mathrm{w}, i}$, covering a range of several minutes to several hours, as a function of the vapor accommodation coefficient on the chamber wall $\left(\alpha_{\mathrm{w}, i}\right)$. The region to the left of the white solid line is that in which $\tau_{\mathrm{g} / \mathrm{w}, i}$ and $\tau_{\mathrm{g} / \mathrm{p}, i}$ are competitive. For low $\alpha_{\mathrm{w}, i}\left(\right.$ e.g., $\left.<10^{-8}\right), \tau_{\mathrm{g} / \mathrm{w}, i}$ is comparable to $\tau_{\mathrm{g} / \mathrm{p}, i}$ only if the vapor has a low accommodation coefficient on the particles $\left(\alpha_{\mathrm{p}, i}<10^{-4}\right)$ or if a relatively small concentration of particles is present in the chamber $\left(R_{\mathrm{a}}<10^{-4}\right)$. For $\alpha_{\mathrm{w}, i}>10^{-4}$, $\tau_{\mathrm{g} / \mathrm{w}, i}$ is estimated to be of the order of several minutes and, as a result, vapor transport to particles is suppressed by competition with the chamber wall, even with perfect particle accommodation $\left(\alpha_{\mathrm{p}, i}=1\right)$ or high particle concentrations $\left(R_{\mathrm{a}}>10^{-2}\right)$.

Overall, in the region (confined by the white solid and dash lines in Fig. 8) where gas-wall partitioning is competitive with gas-particle partitioning, it is necessary to account for vapor wall deposition when deriving SOA yields from chamber experiments. The theoretical framework developed in this study suggests that the area of this region is ultimately controlled by the accommodation coefficient of organic vapors on particles $\left(\alpha_{\mathrm{p}, i}\right)$ vs. the chamber wall $\left(\alpha_{\mathrm{w}, i}\right)$.

\section{Conclusions}

The wall-induced decay of organic vapors is the result of coupled physical processes involving transport of organic vapors from the well-mixed core of a chamber to its wall by molecular and turbulent diffusion, uptake of organic molecules by the Teflon film, and re-evaporation from the wall. The wall-induced dark decay of 25 intermediate/semi-volatility organic compounds generated from the photochemistry of four parent hydrocarbons was monitored in the Caltech dual $24 \mathrm{~m}^{3}$ FEP Teflon chambers. The extent to which organic vapors and the chamber wall interact was found to be similar in used vs. unused Teflon chambers. Based on this observation, one concludes that the Teflon film itself acts as an effective sorption medium, and organic materials deposited from past chamber experiments, if they indeed exist, do not significantly impact the sorption behavior of organic molecules. Reversibility in gas-wall partitioning was observed: evaporation of all 25 compounds that had deposited on the wall 
during an $18 \mathrm{~h}$ deposition period occurred when the chamber temperature was increased from 25 to $45^{\circ} \mathrm{C}$.

Based on a derived model that describes the dynamics of vapor deposition on the chamber wall, a single parameter, the accommodation coefficient $\left(\alpha_{\mathrm{w}, i}\right)$, emerges to govern the extent of the vapor-wall mass transfer process. Moreover, $\alpha_{\mathrm{w}, i}$ exhibits a strong dependence on the molecular properties, such as vapor pressure and oxidation state, of the 25 organics studied. We present an empirical expression for $\alpha_{\mathrm{w}, i}$ as a function of the compound vapor pressure, thus affording the possibility to predict the wall deposition rate of intermediate/semi/non-volatility compounds in a Teflon chamber based on their molecular constituency.

Previous studies have observed the chemical transformation of $\delta$-hydroxycarbonyls to substituted dihydrofurans on the chamber wall (Lim and Ziemann, 2005, 2009; Zhang et al., 2014b), suggesting the potential occurrence of heterogeneous reactions on the chamber wall surface. While the extent to which heterogeneous transformations proceed can be potentially represented through the accommodation coefficient, the occurrence of wall-induced chemistry adds another dimension of complexity in predicting vapor wall deposition rates.
Quantifying the impact of vapor wall deposition on the chamber-derived SOA yield is the next step in assessing the effect of vapor wall deposition of SOA formation and evolution. Future studies will be directed at (1) experiments to determine the accommodation coefficients of organic vapors on particles for a variety of SOA systems, and (2) state-of-art SOA predictive models that describe the dynamics of vaporwall and vapor-particle interactions to estimate the fraction of organic vapor fluxes transported to the suspended particles vs. the chamber wall. 


\section{Appendix A}

\begin{tabular}{|c|c|}
\hline$A\left(\mathrm{~m}^{2}\right)$ : & Total surface area of the chamber wall \\
\hline$\alpha_{\mathrm{p}, i}($ dimensionless): & Accommodation coefficient of organic vapor $i$ on particles \\
\hline$\alpha_{\mathrm{w}, i}$ (dimensionless): & Accommodation coefficient of organic vapor $i$ on the chamber wall \\
\hline$C_{0, i}\left(\mathrm{~g} \mathrm{~m}^{-3}\right)$ & Concentration of organic vapor $i$ over the gas-wall interface \\
\hline$C_{i}^{*}\left(\mathrm{~g} \mathrm{~m}^{-3}\right)$ & Saturation concentration of organic vapor $i$ \\
\hline $\bar{C}_{\mathrm{tot}, i}\left(\mathrm{~g} \mathrm{~m}^{-3}\right)$ & Total concentration of organic vapor $i$ in the chamber \\
\hline $\bar{C}_{\mathrm{v}, i}\left(\mathrm{~g} \mathrm{~m}^{-3}\right)$ & Concentration of organic vapor $i$ in the well-mixed core of the chamber \\
\hline$C_{\mathrm{v}, i}\left(\mathrm{~g} \mathrm{~m}^{-3}\right)$ & Local concentration of organic vapor $i$ in the boundary layer adjacent to the wall \\
\hline $\bar{C}_{\mathrm{w}, i}\left(\mathrm{~g} \mathrm{~m}^{-3}\right)$ & Concentration of organic vapor $i$ that has accumulated on the chamber wall \\
\hline$C_{\mathrm{W}}\left(\mathrm{g} \mathrm{m}^{-3}\right)$ & Equivalent mass of absorbing organic material on the chamber wall \\
\hline $\bar{D}_{\mathrm{p}}(\mathrm{m}):$ & Number mean particle diameter \\
\hline$D_{\mathrm{e}}\left(\mathrm{m}^{2} \mathrm{~s}^{-1}\right)$ : & Eddy diffusivity \\
\hline$D_{i}\left(\mathrm{~m}^{2} \mathrm{~s}^{-1}\right):$ & Molecular diffusivity of organic vapor $i$ \\
\hline$\delta(\mathrm{m}):$ & Thickness of the boundary layer adjacent to the wall \\
\hline$H_{i}$ (dimensionless): & Henry's law constant of organic compound $i$ \\
\hline$J_{\mathrm{v}, i}\left(\mathrm{~g} \mathrm{~m}^{-2} \mathrm{~s}^{-1}\right)$ & Vapor flux arriving at the gas-wall interface \\
\hline$J_{\mathrm{W}, i}\left(\mathrm{~g} \mathrm{~m}^{-2} \mathrm{~s}^{-1}\right)$ & Vapor flux evaporating from the wall \\
\hline$K_{\mathrm{e}}\left(\mathrm{s}^{-1}\right)$ & Eddy diffusion coefficient \\
\hline$K_{\mathrm{W}, i}\left(\mathrm{~m}^{3} \mathrm{~g}^{-1}\right)$ & Gas-wall partitioning coefficient \\
\hline$k_{\mathrm{W}, \operatorname{depo}, i}\left(\mathrm{~s}^{-1}\right)$ : & Deposition rate coefficient to the wall \\
\hline$k_{\mathrm{W}, \mathrm{evap}, i}\left(\mathrm{~s}^{-1}\right)$ : & Evaporation rate coefficient from the wall \\
\hline $\bar{M}_{\mathrm{W}}\left(\mathrm{g} \mathrm{mol}^{-1}\right)$ & Average molecular weight of the absorbing organic material on the wall \\
\hline$N_{\mathrm{p}}\left(\mathrm{m}^{-3}\right)$ & Total number concentration of suspended particles \\
\hline$p_{\mathrm{L}, i}^{0}(\mathrm{~atm}):$ & Vapor pressure of organic compound $i$ as a liquid \\
\hline$\gamma_{i}$ (dimensionless): & Activity coefficient in the wall layer on a mole fraction basis \\
\hline $\bar{v}_{i}\left(\mathrm{~ms}^{-1}\right)$ : & Mean thermal speed \\
\hline$V\left(\mathrm{~m}^{3}\right)$ & Total volume of the chamber \\
\hline
\end{tabular}




\section{The Supplement related to this article is available online at doi:10.5194/acp-15-4197-2015-supplement.}

Acknowledgements. This study was supported by NOAA Climate Program Office AC4 program, award \# NA13OAR4310058 and State of California Air Resources Board agreement 13-321.

Edited by: V. F. McNeill

\section{References}

Barrer, R. M., Barrie, J. A., and Slater, J.: Sorption and diffusion in ethyl cellulose. Part III. Comparison between ethyl cellulose and rubber, J. Polym. Sci., 27, 177-197, 1958.

Compernolle, S., Ceulemans, K., and Müller, J.-F.: EVAPORATION: a new vapour pressure estimation methodfor organic molecules including non-additivity and intramolecular interactions, Atmos. Chem. Phys., 11, 9431-9450, doi:10.5194/acp-119431-2011, 2011.

Corner, J. and Pendlebury, E. D.: The coagulation and deposition of a stirred aerosol, P. Phys. Soc. Lond. B., 64, 645-654, 1951.

Crounse, J. D., McKinney, K. A., Kwan, A. J., and Wennberg, P. O.: Measurement of gas-phase hydroperoxides by chemical ionization mass spectrometry, Anal. Chem., 78, 6726-6732, 2006.

Crump, J. G. and Seinfeld, J. H.: Turbulent deposition and gravitational sedimentation of an aerosol in a vessel of arbitrary shape, J. Aerosol. Sci., 12, 405-415, 1981.

Donahue, N. M., Epstein, S. A., Pandis, S. N., and Robinson, A. L.: A two-dimensional volatility basis set: 1. organic-aerosol mixing thermodynamics, Atmos. Chem. Phys., 11, 3303-3318, doi:10.5194/acp-11-3303-2011, 2011.

Eddingsaas, N. C., Loza, C. L., Yee, L. D., Seinfeld, J. H., and Wennberg, P. O.: $\alpha$-pinene photooxidation under controlled chemical conditions - Part 1: Gas-phase composition in low- and high- $\mathrm{NO}_{\mathrm{x}}$ environments, Atmos. Chem. Phys., 12, 6489-6504, doi:10.5194/acp-12-6489-2012, 2012.

Fahnestock, K. A. S., Yee, L. D., Loza, C. L., Coggon, M. M., Schwantes, R., Zhang, X., Dalleska, N. F., and Seinfeld, J. H.: Secondary Organic Aerosol Composition from $\mathrm{C}_{12}$ Alkanes, J. Phys. Chem. A., published online, doi:10.1021/jp501779w, 2014.

Grosjean, D.: Wall loss of gaseous-pollutants in outdoor Teflon chambers, Environ. Sci. Technol., 19, 1059-1065, 1985.

Kanehashi, S. and Nagai, K.: Analysis of dual-mode model parameters for gas sorption in glassy polymers, J. Membrane. Sci., 253, 117-138, 2005.

Kim, C. S. and Smith, T. L.: An improved method for measuring the thermal coefficient of linear expansion of flexible polymer-films, J. Polym. Sci. Pol. Phys., 28, 2119-2126, 1990.

Kokkola, H., Yli-Pirilä, P., Vesterinen, M., Korhonen, H., Keskinen, H., Romakkaniemi, S., Hao, L., Kortelainen, A., Joutsensaari, J., Worsnop, D. R., Virtanen, A., and Lehtinen, K. E. J.: The role of low volatile organics on secondary organic aerosol formation, Atmos. Chem. Phys., 14, 1689-1700, doi:10.5194/acp-14-1689$2014,2014$.
Lim, Y. B. and Ziemann, P. J.: Products and mechanism of secondary organic aerosol formation from reactions of $n$-alkanes with $\mathrm{OH}$ radicals in the presence of $\mathrm{NO}_{x}$, Environ. Sci. Technol., 39, 9229-9236, 2005.

Lim, Y. B. and Ziemann, P. J.: Effects of molecular structure on aerosol yields from $\mathrm{OH}$ radical-initiated reactions of linear, branched, and cyclic alkanes in the presence of $\mathrm{NO}_{x}$, Environ. Sci. Technol., 43, 2328-2334, 2009.

Loza, C. L., Chan, A. W. H., Galloway, M. M., Keutsch, F. N., Flagan, R. C., and Seinfeld, J. H.: Characterization of vapor wall loss in laboratory chambers, Environ. Sci. Technol., 44, 5074-5078, 2010.

Loza, C. L., Craven, J. S., Yee, L. D., Coggon, M. M., Schwantes, R. H., Shiraiwa, M., Zhang, X., Schilling, K. A., Ng, N. L., Canagaratna, M. R., Ziemann, P. J., Flagan, R. C., and Seinfeld, J. H.: Secondary organic aerosol yields of 12-carbon alkanes, Atmos. Chem. Phys., 14, 1423-1439, doi:10.5194/acp-14-14232014, 2014.

Matsunaga, A. and Ziemann, P. J.: Gas-wall partitioning of organic compounds in a Teflon film chamber and potential effects on reaction product and aerosol yield measurements, Aerosol. Sci. Tech., 44, 881-892, 2010.

McMurry, P. H. and Grosjean, D.: Gas and aerosol wall losses in Teflon film smog chambers, Environ. Sci. Technol., 19, 11761182, 1985.

Meares, P.: The diffusion of gases through polyvinyl acetate, J. Am. Chem. Soc., 76, 3415-3422, 1954.

Michaels, A. S., Barrie, J. A., and Vieth, W. R.: Solution of gases in polyethylene terephthalate, J. Appl. Phys., 34, 1-13, 1963.

Pankow, J. F. and Asher, W. E.: SIMPOL.1: a simple group contribution method for predicting vapor pressures and enthalpies of vaporization of multifunctional organic compounds, Atmos. Chem. Phys., 8, 2773-2796, doi:10.5194/acp-8-27732008, 2008.

Paterson, R., Yampol'skii, Y., Fogg, P. G. T., Bokarev, A., Bondar, V., Ilinich, O., and Shishatskii, S.: IUPAC-NIST solubility data series 70. Solubility of gases in glassy polymers, J. Phys. Chem. Ref. Data, 28, 1255-1450, 1999.

Paul, D. R.: Gas sorption and transport in glassy-polymers, Ber. Bunsen. Phys. Chem., 83, 294-302, 1979.

Paulot, F., Crounse, J. D., Kjaergaard, H. G., Kroll, J. H., Seinfeld, J. H., and Wennberg, P. O.: Isoprene photooxidation: new insights into the production of acids and organic nitrates, Atmos. Chem. Phys., 9, 1479-1501, doi:10.5194/acp-9-1479-2009, 2009.

Sada, E., Kumazawa, H., Xu, P., and Nishigaki, M.: Mechanism of gas permeation through glassy polymer-films, J. Membrane Sci., 37, 165-179, 1988.

Seinfeld, J. H. and Pandis, S. N.: Atmospheric chemistry and physics : from air pollution to climate change (2nd Edn.), John Wiley \& Sons, Inc., Hoboken, NJ, 2006.

Tsujita, Y.: Gas sorption and permeation of glassy polymers with microvoids, Prog. Polym. Sci., 28, 1377-1401, 2003.

Vieth, W. R., Tam, P. M., and Michaels, A. S.: Dual sorption mechanisms in glassy polystyrene, J. Colloid. Interf. Sci., 22, 360-370, 1966.

Yee, L. D., Craven, J. S., Loza, C. L., Schilling, K. A., Ng, N. L., Canagaratna, M. R., Ziemann, P. J., Flagan, R. C., and Seinfeld, J. H.: Secondary organic aerosol formation from low- $\mathrm{NO}_{x}$ photooxidation of dodecane: Evolution of multigeneration gas- 
phase chemistry and aerosol composition, J. Phys. Chem. A., 116, 6211-6230, 2012.

Yeh, G. K. and Ziemann, P. J.: Alkyl nitrate formation from the reactions of $\mathrm{C} 8-\mathrm{C} 14 \mathrm{n}$-alkanes with $\mathrm{OH}$ radicals in the presence of NOx: meausred yields with essential corections for gas-wall partitioning, J. Phys. Chem. A, 118, 8147-8157, 2014.

Zhang, X. and Seinfeld, J. H.: A functional group oxidation model (FGOM) for SOA formation and aging, Atmos. Chem. Phys., 13, 5907-5926, doi:10.5194/acp-13-5907-2013, 2013.
Zhang, X., Cappa, C. D., Jathar, S. H., McVay, R. C., Ensberg, J. J., Kleeman, M. J., and Seinfeld, J. H.: Influence of vapor wall loss in laboratory chambers on yields of secondary organic aerosol, P. Natl. Acad. Sci. USA, 111, 5802-5807, 2014a.

Zhang, X., Schwantes, R. H., Coggon, M. M., Loza, C. L., Schilling, K. A., Flagan, R. C., and Seinfeld, J. H.: Role of ozone in SOA formation from alkane photooxidation, Atmos. Chem. Phys., 14, 1733-1753, doi:10.5194/acp-14-1733-2014, 2014 b. 